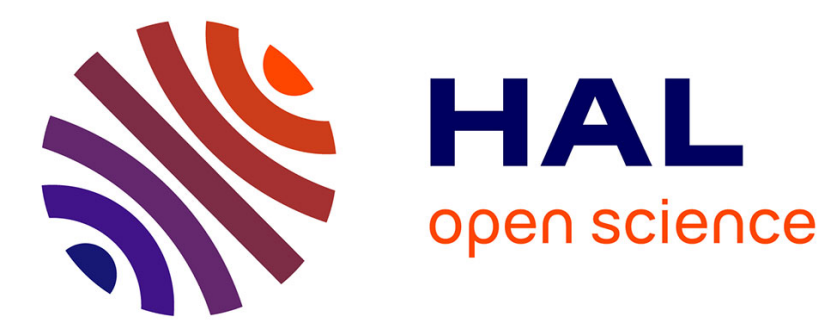

\title{
Découverte d'une nécropole gallo-romaine à Apt (Vaucluse)
}

\author{
André Dumoulin
}

\section{To cite this version:}

André Dumoulin. Découverte d'une nécropole gallo-romaine à Apt (Vaucluse). Gallia - Fouilles et monuments archéologiques en France métropolitaine, 1964, 22 (1), pp.87-110. 10.3406/galia.1964.2190 . hal-01934373

\section{HAL Id: hal-01934373 https://hal.science/hal-01934373}

Submitted on 25 Feb 2020

HAL is a multi-disciplinary open access archive for the deposit and dissemination of scientific research documents, whether they are published or not. The documents may come from teaching and research institutions in France or abroad, or from public or private research centers.
L'archive ouverte pluridisciplinaire HAL, est destinée au dépôt et à la diffusion de documents scientifiques de niveau recherche, publiés ou non, émanant des établissements d'enseignement et de recherche français ou étrangers, des laboratoires publics ou privés.

\section{(ㅇ)(1) $\$$}

Distributed under a Creative Commons Attribution - NonCommercial - NoDerivatives| 4.0 


\title{
DÉCOUVERTE D'UNE NÉCROPOLE GALLO-ROMAINE A APT (VAUCLUSE)
}

\author{
par André DUMOULIN
}

La ville d'Apt, dont les monuments romains ont disparu, conserve cependant sous son sol de nombreux vestiges de la Cité d'Apta-Julia. On connait maints endroits où il suffirait de creuser la terre pour mettre au jour des restes d'habitations. On sait avec précision où se trouvent deux belles mosaïques : l'une recouverte par un bassin du jardin public, l'autre cachée sous une bascule, et si l'on se hasarde dans quelques caves profondes des vieux quartiers autour de la Cathédrale, on sera surpris de reconnaitre l'infrastructure de constructions romaines à la base des murs épais de ces antiques celliers. Des travaux d'urbanisme exécutés en 1903 au quartier du Clos ont permis de découvrir tout un îlot de la vieille ville'. De nombreuses sépultures ont été de tous temps exhumées aux abords de la voie Domitienne, à son entrée et à sa sortie de la cité ; nous en avons signalé et décrit un certain nombre ${ }^{2}$. Enfin dernièrement une nouvelle trouvaille est venue apporter une importante contribution à la connaissance de l'archéologie aptésienne : c'est celle d'un ensemble de 33 tombes appartenant à un vaste cimetière en bordure méridionale de la grande voie romaine, que nous nous proposons d'étudier en détail.

Dans les derniers jours de février 1958, des terrassiers creusaient le sol d'un terrain vague acquis récemment par $M$. Louis Piton, industriel, situé exactement en face de la cour d'entrée de son usine de fruits confits, de l'autre côté du Chemin du Gaz ${ }^{3}$, quartier St-Lazare ${ }^{4}$, en vue d'établir les fondations d'une grande bascule (fig. 1). Dès le début des travaux, quatre amphores contenant plusieurs vases accompagnés d'ossements brûlés furent mises au jour à moins d'un mètre de profondeur et malheureusement brisées par la pioche des manœuvres. Grâce à l'amabilité du propriétaire, que nous remercions vivement ici, nous pûmes sans délai entreprendre des fouilles systématiques pendant plus d'un mois dans la partie du terrain (environ $200 \mathrm{~m}^{2}$ ) susceptible de contenir d'autres

(1) Sur les antiquités de l'ancienne capitale des Vulgientes, voir Fernand SAlve : monographie de La Ville d'Apt (Vaucluse), Apt, 1903 et : Découverles gallo-romaines à Apt, Caen, 1911.

(2) André Dumoulis, Recherches archéologiques dans la région d'Apt, Gallia, XVI-1958, p. 205-215.

(3) Cette voie a actuellement une largeur de $6 \mathrm{~m}$. 50 environ. On sait que hors les villes la largeur de la chaussée des routes romaines paraît avoir varié de 5 à 8 mètres, mais la largeur de $6 \mathrm{~m}$. semble bien avoir été la normale. (Voir Albert Grenier, Manuel Deuxième partie, L'Archéologie du sol; les routes, p. 367).

(4) Parcelle 640, Section D du plan. Dans la cour même de cette usine furent découverts en 1951 et 1956,4 sarcophages en plomb avec riche mobilier funéraire (André Dumoctıs, op. cit., p. 215 à 222). 

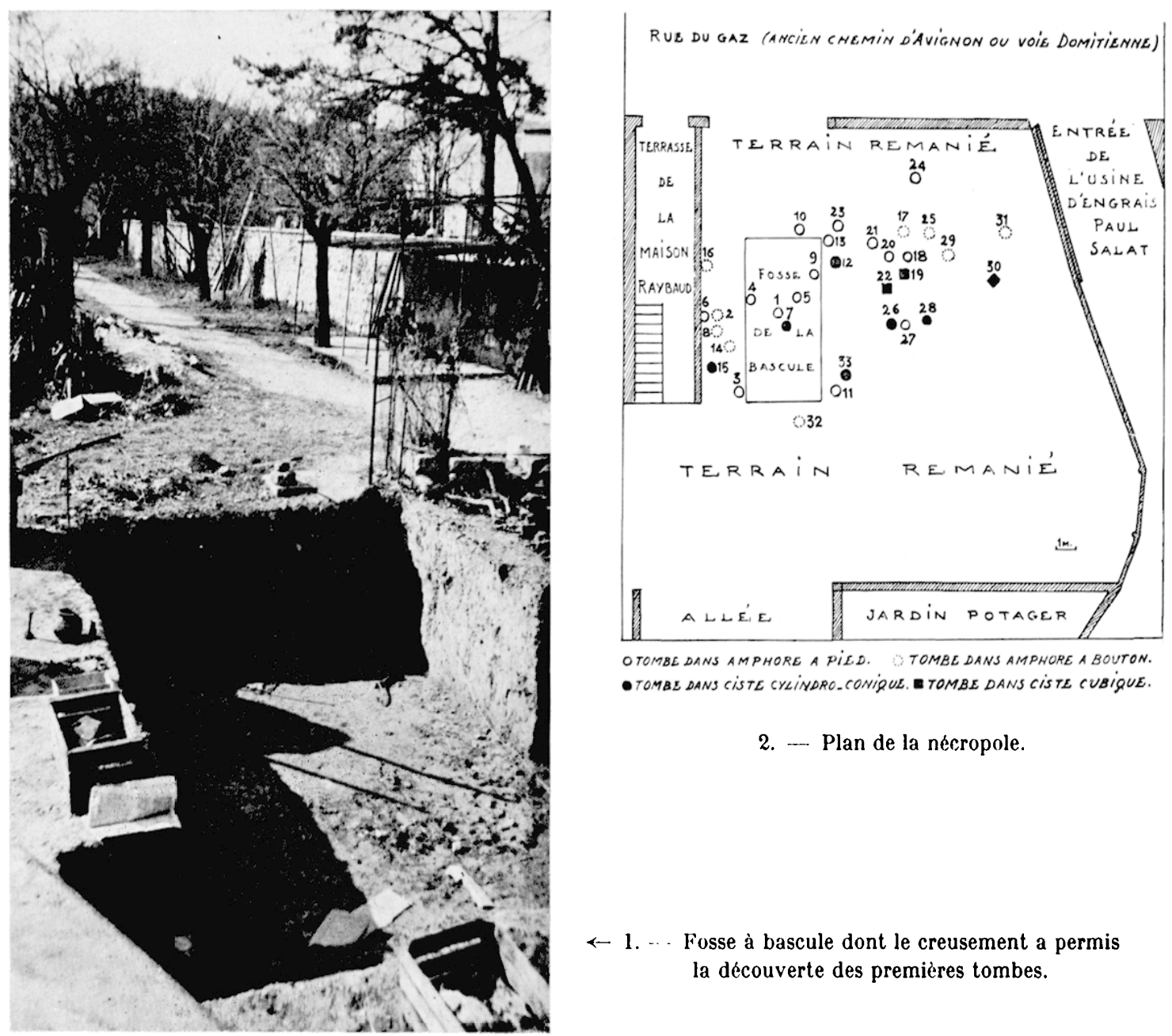

sépultures (fig. 2). Nos espoirs ne furent pas déçus car 27 tombes furent encore découvertes et un important matériel archéologique a été recueilli.

Toutes à incinération, les sépultures se composent individuellement :

$1^{\circ}$ d'une partie externe qui est le contenant de la tombe : soit une amphore complète mais dont la panse a été découpée tout autour vers le haut, sous les anses, afin de permettre l'insertion du mobilier funéraire, soit un ciste ou caisson en pierre, avec opercule ;

$2^{\circ}$ d'une partie interne comprenant l'urne cinéraire où ont été déposés les restes calcinés du défunt, accompagnée, sauf exception, d'un mobilier funéraire d'importance variable. Dans certains cas, faute de place suffisante à l'intérieur du tombeau, un ou plusieurs récipients avaient été mis en dehors : à côté pour les amphores, sur le couvercle lorsqu'il s'agit des cistes.

On peut distinguer 3 types d'amphores :

type I : amphore en terre cuite fine rougeâtre à parois minces épaissies à la base, de forme oblongue, à panse surhaussée et pied circulaire, creux pouvant tenir droit. Le col est court et l'orifice a un rebord plat sur le dessus, arrondi sur les côtés. Deux anses opposées, peu développées, plates, dégagées, joignent le milieu du col et le haut de la panse au-dessus 
de sa plus grande largeur qui se situe à peu près aux $2 / 3$ de la hauteur (type Dressel 30 ). Dimensions moyennes $^{5}$ : H : $61 \mathrm{~cm}-\mathrm{DM}: 42 \mathrm{~cm}-\mathrm{DB}: 13,5 \mathrm{~cm}$ (fig. $\left.3, \mathrm{I}\right)$;

type II : amphore pansue, sphérique, de facture moins soignée que la précédente, en argile marron foncé fine, dure, à parois épaisses amincies vers le bas. Pas de station verticale possible. Le fond est muni en son centre d'une petite protubérance saillant extérieurement. Le col est très court et l'ouverture a un rebord un peu tombant. Les anses, boudinées, petites par rapport à l'ensemble du vaisseau sont placées haut (type Dressel 20, dit type espagnol). Dimensions moyennes : H : $72 \mathrm{~cm}-$ DO : 10,5 cm -DM : $57 \mathrm{~cm}$ (fig. 3, II) ;

type III : assemblage mixte : amphore du type II dont le sommet (col et anses), détaché au préalable, a été remplacé par la partie supérieure d'une autre amphore du type I qui la coiffe en recouvrant de quelques centimètres la bordure découpée (fig. 3, III).

Les tombes dans ciste et cuve de pierre sont de deux sortes :

type A : ciste de forme cylindro-conique, constitué par un bloc de mollasse blanche tendre ou plus rarement de calcaire urgonien, façonné et creusé dans la masse mais laissant un fond épais, et fermé par un couvercle circulaire (fig. 3, A) ;

type B : ciste de forme cubique, de même matière, avec opercule carré à bords souvent chanfreinés (fig. $3, \mathrm{~B}$ ).

Les sépultures se répartissent ainsi :

11 dans amphores de type I

7 dans amphores de type II

2 dans amphores de type III

10 dans cistes de type $\mathrm{A}$

3 dans cistes de type $\mathbf{B}$

C'est l'ordre dans lequel nous allons les décrire en conservant pour chaque série la succession chronologique.

\section{SÉpultures dans amphores du Type I}

\section{Tombe 1}

Découverte par les terrassiers, seule une lampe à huile incomplète en terre cuite rosée a pu être recueillie; elle porte sous le réservoir la marque du fabricant : PVLLI, en relief (type vallée du Pô, forme Dressel 5c), (fig. 8, A).

\section{Tombe 3}

L'urne cinéraire, qui n'a pu être récupérée par les terrassiers, devait être en verre, d'après quelques débris retrouvés.

a) Balsamaire en verre jaunâtre à col long terminé à la base par un corps bulbeux plat en dessous où se trouve estampée la marque du verrier : VALERIANVS (en lettres capitales à extrémités bouletées) disposée en demi-cercle autour d'un sapin stylisée ${ }^{6}$, (fig. 4, B).

b) Dupondius de TRAJAN (98-117 ap. J.-C.), très fruste.

(5) Abréviations : DO : diamètre de l'orifice - DM : diamètre maximum - DB : diamètre de la base ou du pied $-\mathrm{H}$ : hauteur $-\mathrm{L}:$ longueur $-\mathbf{l}$ : largeur.

(6) C'est la forme 25 de Morin-Jean (La Verrerie en Gaule sous l'Empire romain, 1913) qui figure p. 76 une marque de verrier, moulée, voisine de la nôtre. 

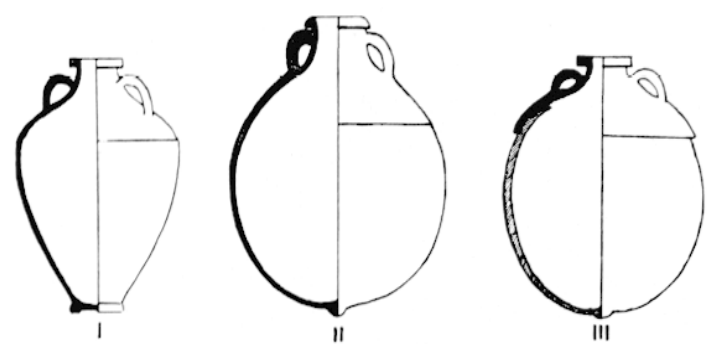

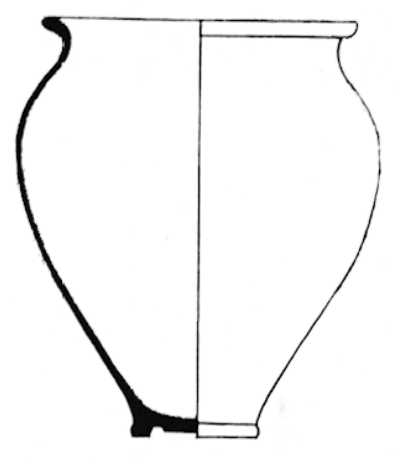

A

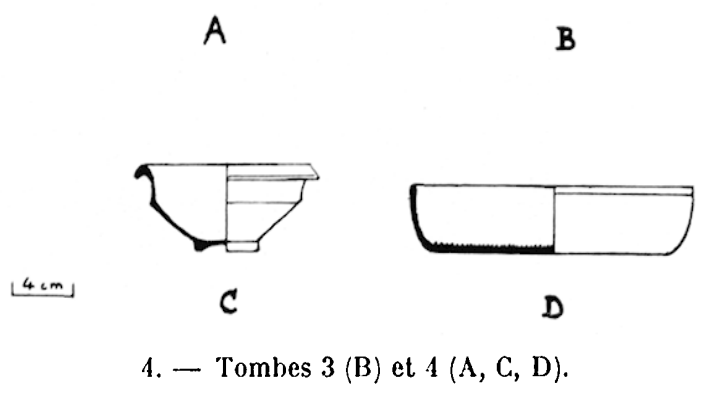

4. - Tombes $3(\mathrm{~B})$ et $4(\mathrm{~A}, \mathrm{C}, \mathrm{D})$.

3. -- Types des amphores et des cistes.

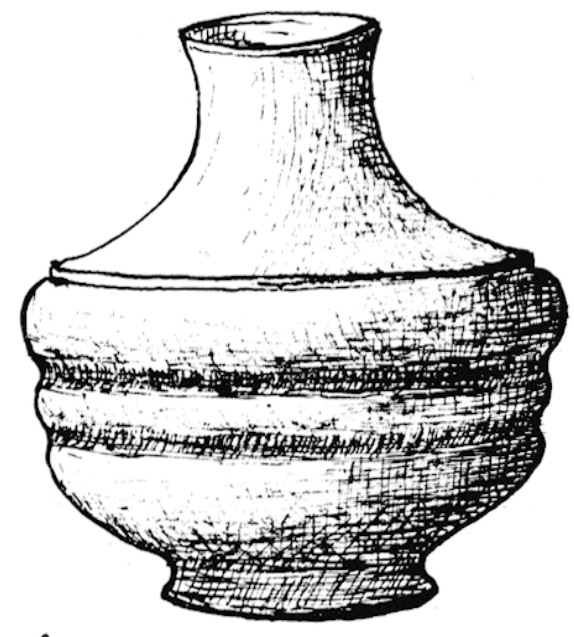

A

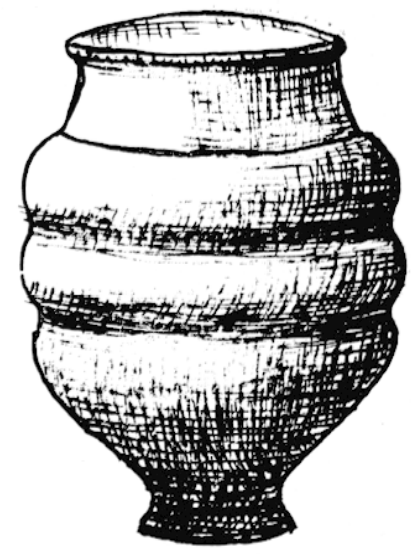

B

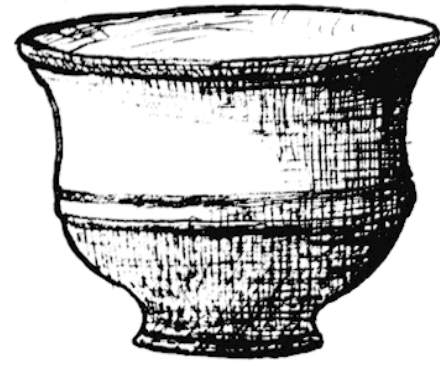

C

5. - Vases à bourrelets de la tombe 4.

\section{Tombe 4}

a) Urne cinéraire en terre cuite dure, grise, peignée horizontalement sur les $2 / 3$ inférieurs, lisse en haut. Bords arrondis col très court et panse amincie à la base terminée par un pied peu élevé circulaire (fig. $4, \mathrm{~A}$ ).

b) 3 récipients : bouleille, vase et bol en terre grise fine pailletée de mica à engobe gris foncé dont l'air de parenté est frappant. La panse de la bouteille est circulaire, large, formée par 3 bourrelets inégaux superposés; le goulot à parois arquées, à orifice sans lèvre, s'élève de la panse par un petit ressaut; le pied est creux, de faible hauteur (fig. $5, \Lambda$ ). Les 3 bourrelets se retrouvent sur le vase terminé en haut par un col large et court, en bas par un petit pied tronconique (fig. 5 , B). Le 

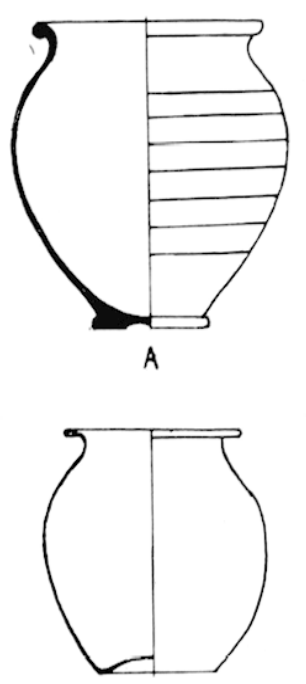

c

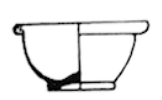

E

6. - Tombes $5(\mathrm{~A}, \mathrm{~B})$ et 6 (C,D,E,F,G).

D

G

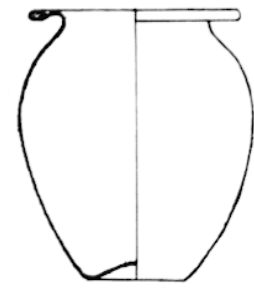

A
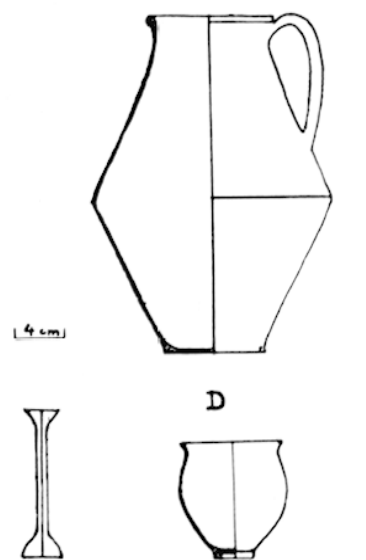

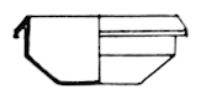

D

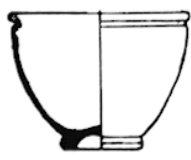

G
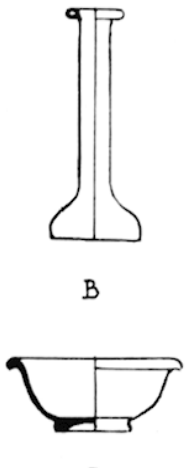

E

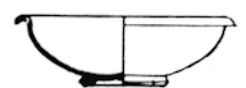

H

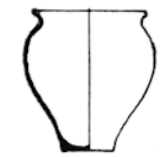

C

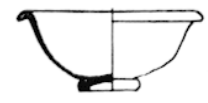

$\mathbf{F}$

bol est évasé, à pied circulaire et parois minces; un fin bourrelet limité par 2 sillons fait le tour de la panse à peu près au milieu du récipient (fig. $5, \mathrm{C}$ ).

c) Écuelle en terre commune grise, à bords tombants, panse angulaire et pied bas circulaire (fig. 4, C).

d) Jalle à parois légèrement obliques, peu élevées, à fond circulaire plat présentant intérieurement de nombreux cercles concentriques : terre cuite grise, ordinaire (fig. 4, D).

e) Une trentaine de petits clous en fer de $2 \mathrm{~cm}$ de long. à tête ronde plate.

\section{Tombe 5}

a) Urne cinéraire en terre cuite marron. Forme globuleuse à bords arrondis et panse légèrement cannelée horizontalement (fig. 6, A).

b) Ecuelle en terre commune marron clair ; panse angulaire, bords horizontaux et pied plein (fig. 6, B).

c) Lampe en argile orangée, sans vernis, signée : FORTIS, munie sur le bord de 2 petites protubérances arrondies, à sillon médian et. à l'opposé du bec d'une sorte d'appendice de préhension, creux à l'intérieur, incomplet (type de la vallée du Pò, forme Dressel $5 \mathrm{c}$ à canal ouvert), (fig. 8, B).

\section{Tombe 6}

a) Urne cinéraire en verre bleuté à panse arrondie, fond saillant à l'intérieur du récipient et bords plats ourlés en dedans (forme 1 de Morin-Jean) (fig. 6, C).

b) Petit "lacrymatoire " en verre très fin, col long reliant une base tronconique à une ouver-

(7) Nous conserverons le nom de "lacrymatoire " terme consacré par l'usage, pour désigner ce genre de petit récipient dont le nom exact est : unguentarium, vase servant à contenir des parfums ou des pommades (voir Dicl. des Ant. de Daremberg et Saglio, au mot : unguentarium). 
ture évasée non ourlée (forme 21-25 de Morin Jean). Sous le fond, marque de verrier représentant, dans un grènetis, un motif en relief indéfinissable (fig. 6, F).

c) Bol en verre clair transparent fin ; pieds et bords épaissis formés par un repli de la paraison, à bande périphérique foncée. Fond saillant en cône intérieurement (fig. 6, E).

d) Urnelte en terre cuite rougeâtre à base étroite et épaisse (fig. 6, G).

e) Cruche en argile jaune à panse biconique munie d'une anse plate arrondie réunissant le bord mème au haut de la panse ; ouverture circulaire sans rebord, pied bas, plein (fig. 6, D).

f) Lampe en terre jaune clair très tendre; bec rond à volutes latérales (forme Dressel 11. Fin du ier siècle). Le dessus est orné d'une victoire ailée, drapée, marchant, de face, à la main droite tenant un bouclier rond, la gauche, une palme (fig. 8, D).

g) Dupondius de DOMITIEN (81-96 ap. J.-C.), assez usé, où l'on distingue à l'avers le buste de l'empereur lauré à droite avec la légende : ...CAES DOMI TI AN.AVG.... et au revers un personnage assis à gauche entre $S$ et $\mathrm{C}$. A l'exergue : A V G V S T.

h) 1 clou en fer.

La cruche, la lampe, la pièce de monnaie et le clou se trouvaient à l'extérieur de la tombe.

\section{Tombe 9}

La partie supérieure de l'amphore porte, gravé à la pointe en grandes lettres majuscules : G A M. Le mobilier funéraire, abondant, comprend :

a) Une urne cinéraire globuleuse en verre bleu clair (fig. 7, A).

b) Un balsamaire en verre clair, à panse bulbeuse (fig. 7, B).

c) Une urnelle en terre cuite marron foncé (fig. 7, G).

d) Une écuelle carenée en poterie commune, dure, micacée, à bords très tombants et fond plat (fig. 7, D).

e) 4 récipients en terre fine rouge vernissée (fig. $7, \mathrm{E}, \mathrm{F}, \mathrm{G}, \mathrm{H}$ ) :

101 écuelle à bords lisses (forme Drag. 36).

$2^{\circ} 2$ bols semblables à bords non barbotinés (forme Drag. 35). L'un contient 3 dents humaines : 2 molaires et 1 canine, très usées, et porte sur sa paroi externe les lettres : M I, gravées à la pointe.

$3^{0} 1 \mathrm{bol}$ élevé à parois légèrement convexes, pied haut, profond avec estampille de potier intérieure : O. F. I. R. M. S.

f) 1 lampe en terre cuite jaunâtre avec traces de couverte marron clair. Type du ${ }_{I^{\mathrm{e}}}^{\mathrm{e}}$ siècle à bec court, rond, séparé du réservoir par une incision horizontale (forme Dressel 20, sans anse). Sur le dessus, buste d'Hélios en relief, de face, à 5 rayons partant de la tête ${ }^{8}$,(fig. 9, A).

\section{Tombe 11}

Lrne cinéraire en terre cuite marron clair, à bords arrondis, col court oblique avec léger sillon circulaire (fig. 10, A).

\section{Tombe 13}

Urne cinéraire en argile de même type que la sép. 11 (fig. 10, B).

\section{Tombe 23}

a) Virne cinéraire semblable aux 2 précédentes (fig. 10, C).

b) Écuelle en terre rouge vernissée mate, à bords lisses ; sur la paroi extérieure, graflite en

(8) Des lampes à ornement semblable ont été rencontrées à Glanum (P. de Brun, Les lampes antiques en argile du Musée des Alpilles à Saint-Rémy-de-Provence, B.-du-R., extrait des Cahiers d'Hisloire el d'Archéologie, Nîmes, 1933. No 12 et pl. II, fig. 12), à Vaison (exemplaire à 5 rayons au Musée, à 7 au Musée Calvet d'Avignon et 12 au Musée de St--Germain. J. SAUTEL, Vaison dans l'Anliquité, II. Catalogue des objels romains trouvés à Vaison et duns son territoire 1926, nos 1378 à 1382) et dans la nécropole gallo-romaine de Sannes (Vaucluse), A. Dumoulin, op. cil., p. 234, fig. 40, $n^{\circ} 8$ ). 


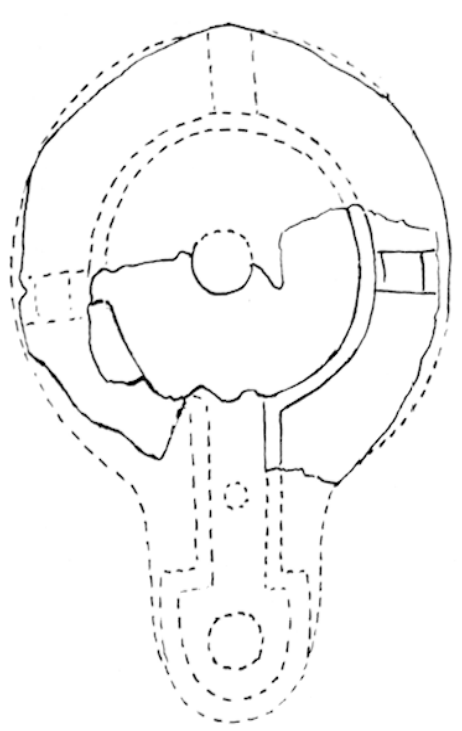

priscs

A
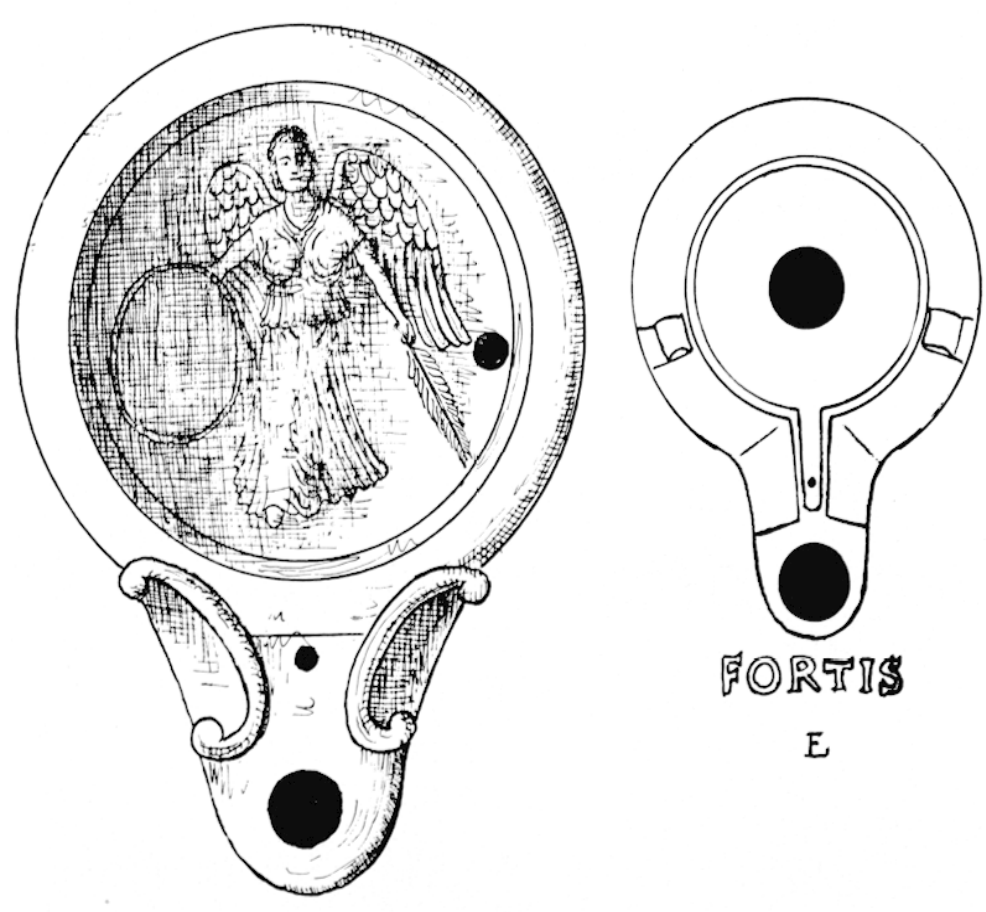

[요제

E

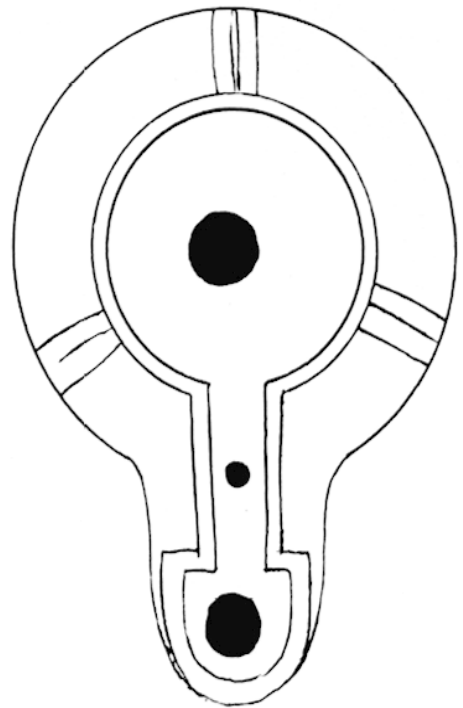

BORTIS

C

D

8. - Lampes des tombes 1 (A), 5 (B), 12 (C), 6 (D). Environ $\frac{2}{3}$ gr. nat. 
lettres cursives : MARGIILLINVS ${ }^{9}$ et, sur le pied, signes géométriques : losange et triangle gravés, de part et d'autre d'une ligne droite (fig. 11).

c) Bol en terre jaunâtre tendre revêtue d'un vernis rouge très écaillé, bord épaissi en bourrelet, col vertical séparé de la panse par un sillon circulaire; pied bas évidé (fig. 10, D).

\section{Tombe 24}

Sur la panse de l'amphore, au-dessus de l'anse est gravé à la pointe le graffite : IIRMIIS, en cursive. A l'intérieur, unique récipient : une urne cinéraire en terre cuite marron patinée, de taille inhabituelle (près de $30 \mathrm{~cm}$ de hauteur), avec quelques ossements brûlés (fig. 12, A).

\section{Tombe 27}

L'urne cinéraire manque. Dans l'amphore se trouvaient :

a) 1 urnetle en poterie commune brun rouge (fig. 12, B).

b) 1 vase à liquide en terre jaune clair, panse ronde égèrement cannelée anse courte et orifice étroit à bord horizontal (fig. 12, C).

A côté de l'amphore, en pleine terre, avaient été disposés 8 récipients en verre dont 6 appartiennent à la série des verreries imitant la forme des bols et écuelles en terre rouge vernissée des potiers romains des $1^{\text {cr }}$ el ${ }_{11}$ e siècles de notre ère (Drag. 35 et 36 ).

c) 2 écuelles en verre blanchâtre (f. 91 de Morin-Jean), identiques, basses à pied large (f. Drag. 36 ), (fig. 12, D).

d) 1 bol en verre de même teinte que les 2 écuelles précédentes, un peu plus haut de panse et de pied (forme Drag. 7) (fig. 12, E). $12, \mathrm{~F})$.

e) 2 petits bols identiques en verre verdâtre à bordure plus foncée (forme Drag. 35), (fig.

f) 1 bol de même forme que les 2 précédents à bord lisse en dessus avec un filet saillant en dessous (fig. 12, G).

g) 1 carafon à parois très minces $(1 / 10 \mathrm{de} \mathrm{mm})$ transparentes ; goulot étranglé à la base, évasé à l'ouverture (fig. 12, H).

h) 1 verre à boire oblong à pied circulaire épais (incomplet).

i) Enfin 3 aiguilles identiques, en os, de $12 \mathrm{~cm}$ de longueur, à chas large el allongé surmonté d'une extrémité conique ${ }^{10}$, complétaient cet important ensemble funéraire.

\section{Sépultures dans amphores du Type II}

Les tombes 2 et 14 étaient représentées seulement par une amphore vidée de son contenu.

\section{Tombe 16}

Découverte au ras des fondations d'un mur, elle avait été détériorée lors de la construction de celui-ci. Nous avons pu en retirer, tout en morceaux :

a) Une urnetle en terre cuite marron clair (fig. 13, A).

b) Un petit vase globuleux en terre friable ocre jaune avec anse fine incomplète (fig. 13, B).

c) Une lampe en argile tendre blanchâtre à bec court (II ${ }^{\mathrm{e}}$ siècle) avec couvercle orné : aigle de face aux ailes éployées, la tète à droite ${ }^{11}$ (fig. 9, G).

(9) Nom connu à Glanum sur un autel dédié à Hercule, avec la même graphie (II pour E) H. RoLtavo, Fouilles de Glanum 1947-1956, XI e supplément à Gallia, 1958, p. 111 et pl. 41, n². L'auteur note que "le cognomen Marcellinus apparait 22 fois dans le C.I.L.XII. "

(10) Ces aiguilles sont semblables à celles figurées par VAssY et MüL.LER : Ebauches d'objels gallo-romains en os de Sainle-Colombe près Vienne (Isère), Rhodania, $4^{\mathrm{c}}$ année, 1922, pl. II, nos 2 et 3 ).

(11) Des lampes de ce genre ont été trouvées à Vaison (J. Sactes, op. cil., nos 1493 et 1494, p. 440), à Glanum (P. de Brun, op. cil., no 65, pl. V'I, fig. 8). Le Musée d'Avignon en possède une, sans provenance, dans la collection Calvet (P. de Brun et S. Gagnière. Les lampes antiques du Musée Calvet d'Avignon. Carpentras, Batailler, 1937, p. 37, no 136 et pl. IX!. 

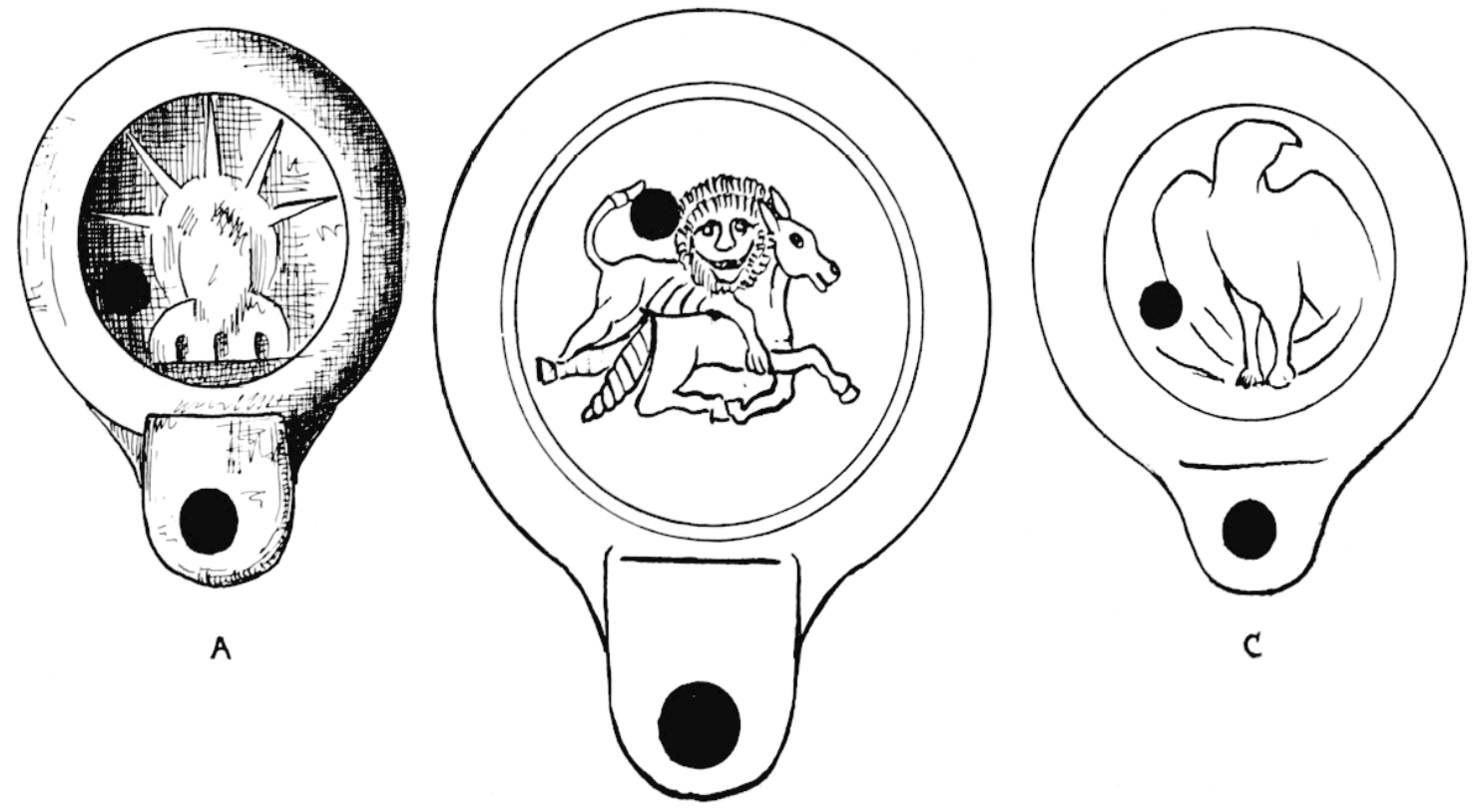

B

9. - Lampes des tombes 9 (A), 28 (B) et 16 (C). Environ $\frac{2}{3}$ gr. nat.
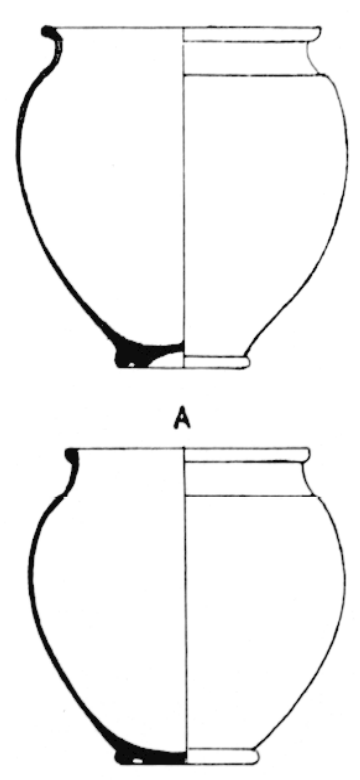

C

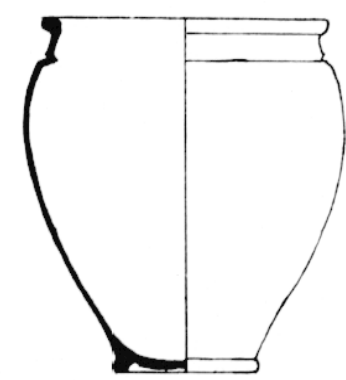

B

$4 . m$

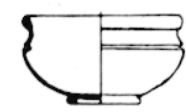

D

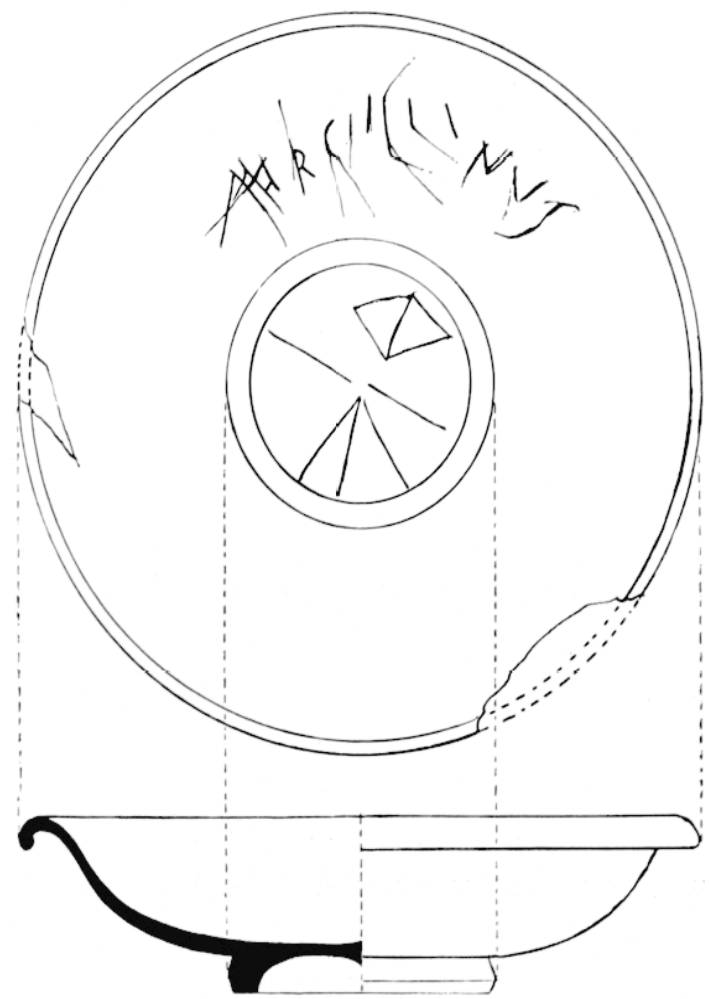

10. - Tombes $11(\mathrm{~A}), 13(\mathrm{~B})$ et 23 (C,D).

11. - Écuelle rouge vernissée de la tombe 23 avec le graffite : MARCIILINVS. D : $18 \mathrm{~cm}$. 
d) 4 récipients : 2 bols et 2 écuelles qui ont comme caractères communs d'être en verre transparent très fin, d'avoir les bords et le pied constitués par un repli de la paraison, le fond épaissi au centre et renflé à l'intérieur ${ }^{12}$ (fig. $13, \mathrm{C}, \mathrm{D}, \mathrm{E}, \mathrm{F}$ ).

\section{Tombe 25}

a) Urne cinéraire à large rebord, col dégagé et panse globuleuse en verre bleu clair (fig. 14, A).

b) Grand balsamaire en verre laiteux, très mince, à long col étranglé à sa base, panse circulaire large et écrasée (fig. 14, B).

c) Amphorisque en verre opalin à base pointue. C'est une gracieuse petite ampoule munie de 2 anses courtes en verre bleu foncé (forme 29 de Morin-Jean ; rare), (fig. 14, C).

d) Flacon en verre réduit en menus morceaux, fond plat, 2 anses bleu foncé (fig. 14, D ; reconstitution).

e) Petite lampe en bronze à bec allongé, dessus plat uni à trou central et anse horizontale (fig. 14, E).

f) 13 petits objets en os appartenant sans doute à un même ensemble (jeu?) (fig. 16) :

4 disques plats perforés au centre d'un trou de grandeur inégale,

7 tiges courtes tournées et façonnées avec renflements moulurés, toutes dissemblables sauf les 2 plus petites,

2 cylindres creux respectivement de $2,7 \mathrm{~cm}$ et $1,5 \mathrm{~cm}$ de long, $1,1 \mathrm{~cm}$ et $1 \mathrm{~cm}$ de diamètre.

g) Enfin le tamisage de la terre contenu dans cette tombe a fait apparaître quelques menus fragments de raclure d'or, vestiges probables de la dorure d'un des récipients en verre du mobilier funéraire.

\section{Tombe 29}

a) Urne cinéraire en verre, de type habituel (fig. 15, D).

b) Petit vase en terre commune marron foncé (fig. 15, E).

c) Longue bouteille en verre verdâtre zoné à parois épaisses, bords horizontaux ourlés en dehors, col court et panse allongée s'évasant vers la base (fig. 15, F).

d) Aiguille en os très fragmentée.

Tombes 31 et 32

Amphores vidées de leur contenu.

\section{SÉPUltures DaNS AMPhORES DU TyPE III}

\section{Tombe 8}

a) Grande urne cinéraire en verre bleu-vert, globuleuse (fig. 15, B).

b) Balsamaire en verre bleu clair, haut col à bords ourlés en dehors, panse à profil " en forme de doucine " ítype 23 de Morin-Jean) et fond légèrement concave (fig. 15, C).

c) 2 clous en fer.

\section{Tombe 17}

Amphore vidée de son contenu. - La partie supérieure porte un graffite au col : PASS sous un grand signe tectiforme.

(12) Ce type de récipient en verre a déjà été trouvé à Apt, en 1848 dans une sépulture gallo-romaine (exemplaire donné au Musée Calvet en 1849. Catalogue manuscrit du Musée Calvet, p. 479, nº 152 A. 


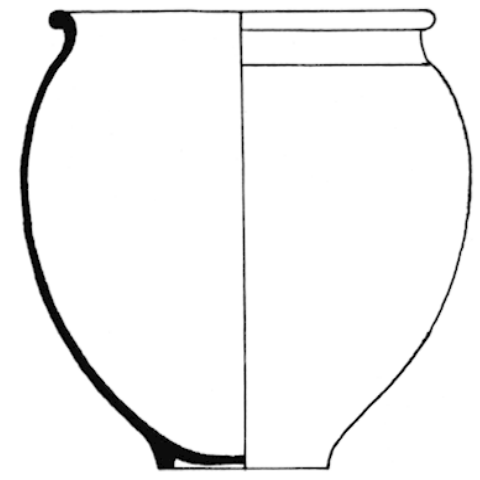

A
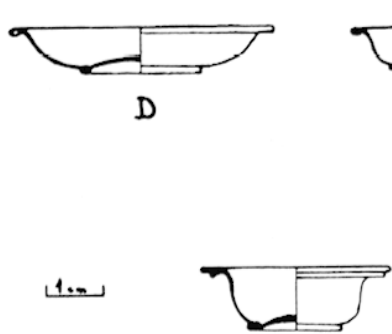

o

12. - Tombes $24 \mathrm{c} \mathrm{A})$ et 27 (B, C,D,E,F,G,H).

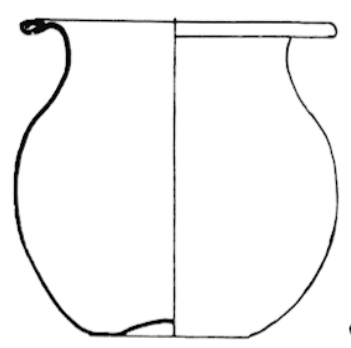

A

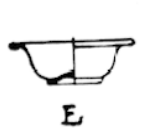

E

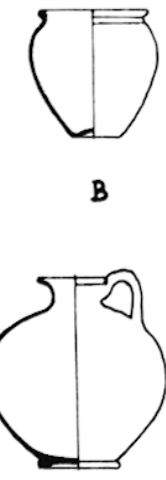

C
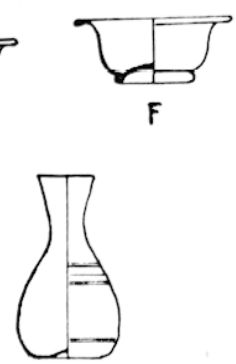

$\mathrm{H}$

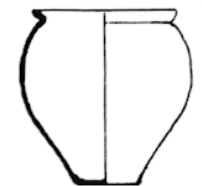

$A$
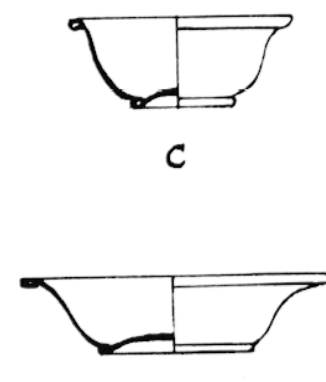

E

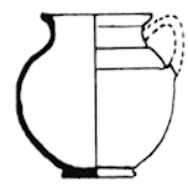

B

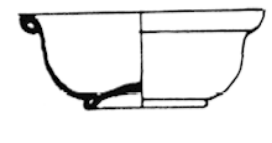

D

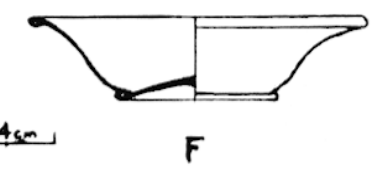

13. - Tombe 16.
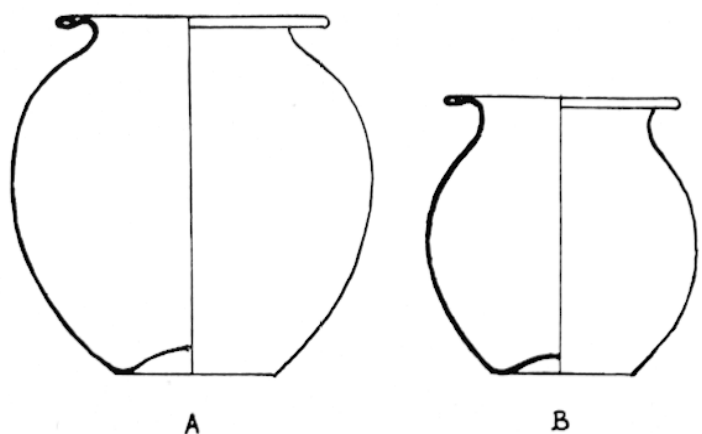

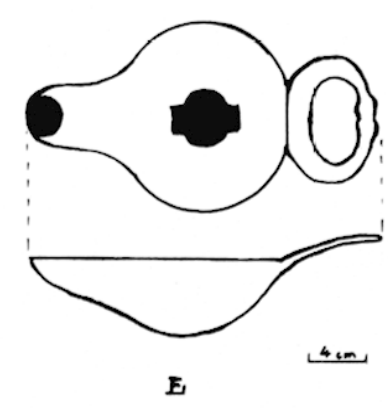

14. - Tombe 25.

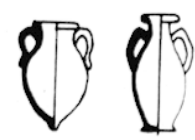

C D

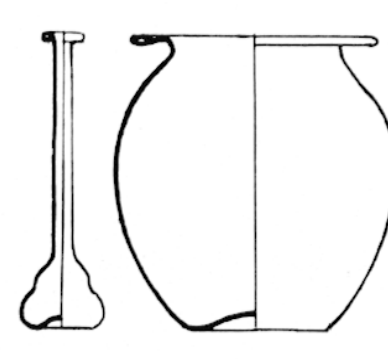

D

15. - Tombes $7(\mathrm{~A}), 8(\mathrm{~B}, \mathrm{C})$ et $29(\mathrm{D}, \mathrm{E}, \mathrm{F})$.

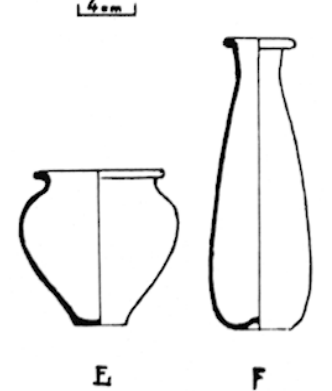



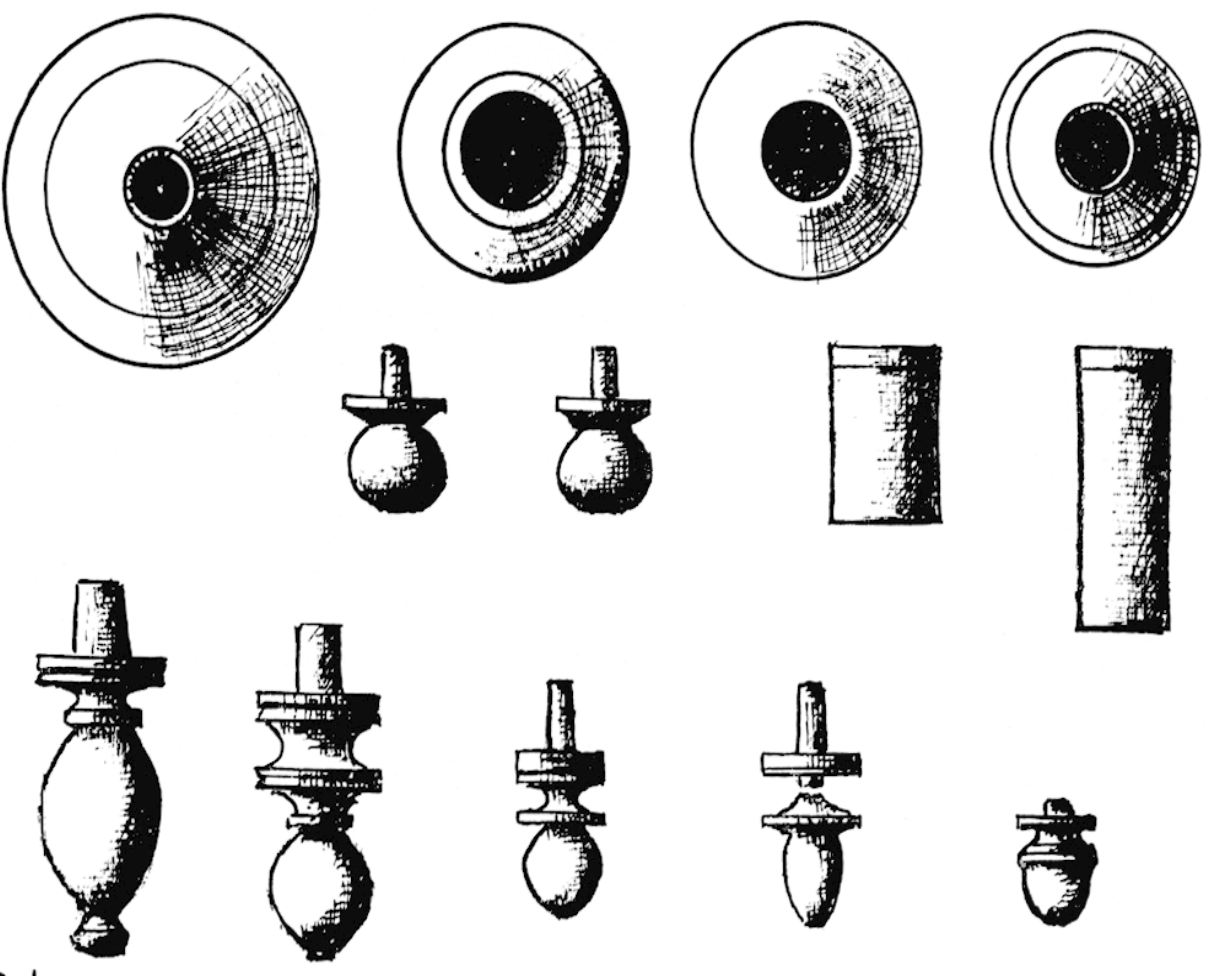

$1 \mathrm{~cm}$

16. - Tombe 25. Objets en os.

\section{Sépultures dans cistes du Type A}

\section{Tombe 7}

Elle ne contenait qu'une urne cinéraire en verre de forme ordinaire (fig. 15, A).

\section{Tombe 10}

a) Lirne cinéraire en verre bleuâtre, sphérique, à bords larges et plats (fig. $19, \Lambda$ ).

b) Balsamaire en verre jaunâtre à panse bulbeuse (f. 25 de Morin-Jean). Sous le fond, marque de verrier dans un rectangle : MP, en caractères de $1,5 \mathrm{~cm}$ de hauteur (fig. 19, B).

c) Vase en verre opalin, cylindrique, un peu renflé vers le bas, à pied creux et bords droits; 2 petits filets saillants en font le tour l'un sous le bord, l'autre près de la base (fig. 19, C).

\section{Tombe 12}

a) Lrne cinéraire en verre de forme ovoïde assez élevée (fig. 19, I)). Parmi les ossements calcinés qui l'cmplissaient à moitié se trouvait une valve de coquille marine blanchâtre de la famille des Vénérides ${ }^{13}$.

b) Balsamaire en verre transparent très fin, à panse piriforme et base légèrement concave (fig. 19, E).

c) Fiole en verre jaunâtre ; fond tronconique épaissi (fig. 19, H).

d) Lampe en terre cuite marron clair ; type de la Vallée du Pò, à 3 protubérances sur le bord,

(13) Il s'agit de Merelrix Chione Lam. ou cytherea chione, commune en Méditerranée particulièrement en Roussillon (détermination de M. Léon Germand, conservateur du Musée Requien à Avignon). 

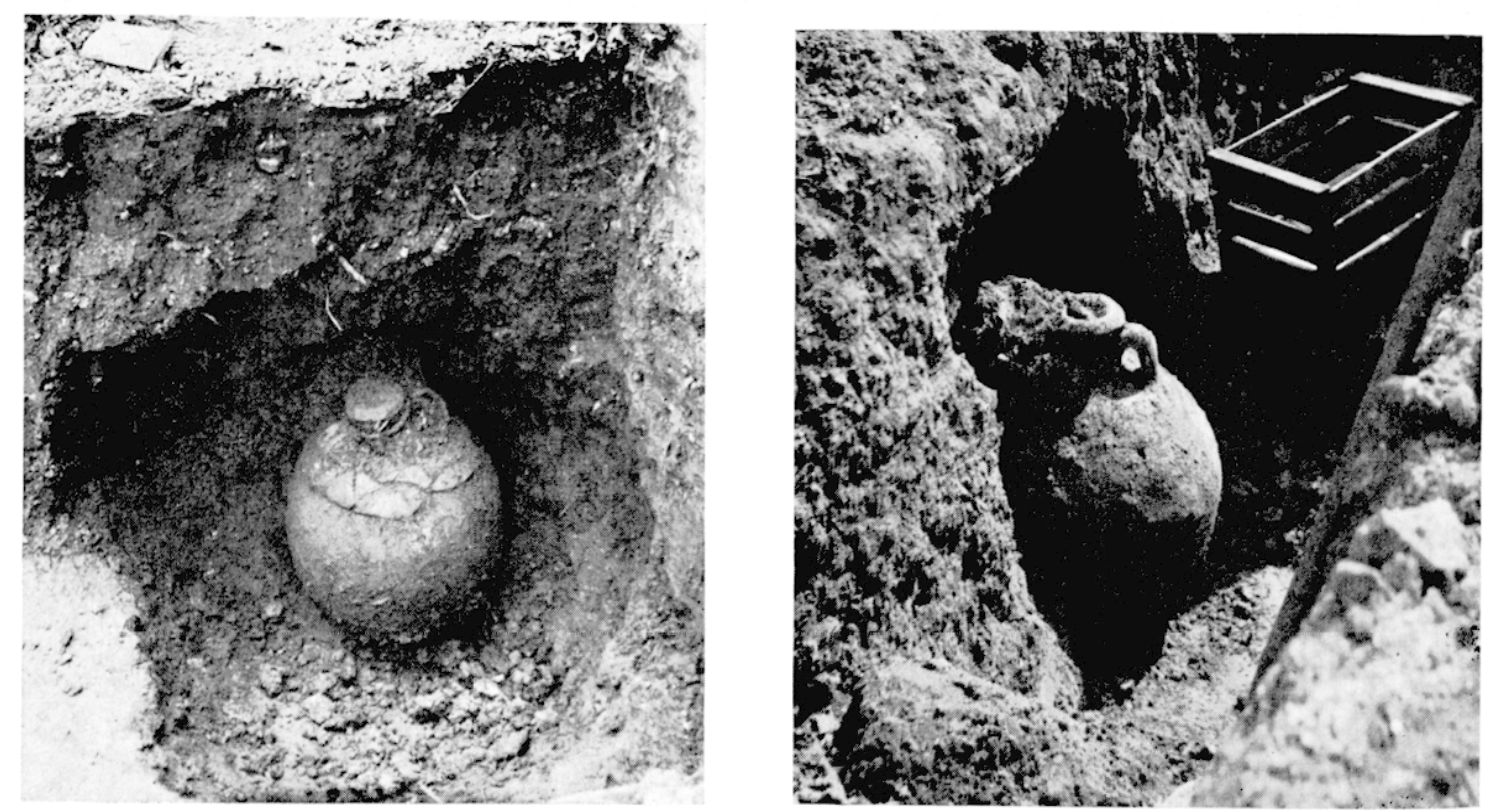

17. - Sépultures dans amphores (tombes 8 et 25).
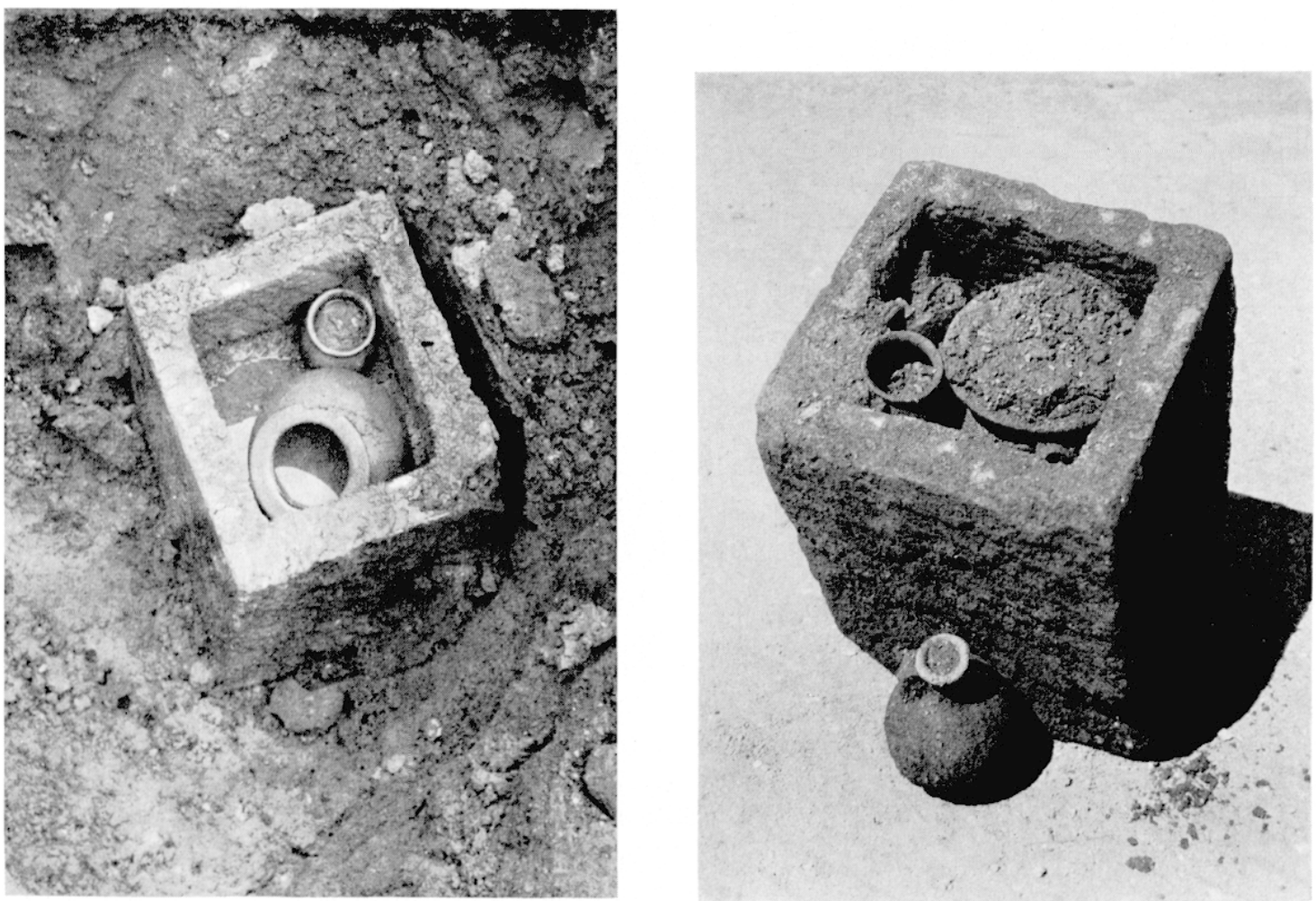

18. -- Sépultures dans cistes cubiques (tombes 19 et 30 ). 
signée : FORTIS (f. Dressel $5 \mathrm{c}$, à canal ouvert) (fig. 8, G). A l'extérieur du ciste, tout à côté, ont été trouvés :

e) une urnette en argile noirâtre (fig. 19, G).

f) un balsamaire en verre à col et à ouverture étrangement larges, pour une panse bulbeuse de dimensions habituelles. Marque de fabricant disposée en cercle sous le fond (fig. 19, F).

\section{Tombe 15}

Ciste brisé dont le mobilier avait disparu.

\section{Tombe 18}

Urne cinéraire en verre transparent (fig. 20, A).

\section{Tombe 20}

a) Urne cinéraire en verre ; forme sphérique à col très court et bords larges ourlés en dedans (fig. 20, B).

b) Petite ampoule piriforme en verre verdâtre, à fond ombiliqué et embouchure débordante ourlée en dedans (forme à rapprocher du bombylios corinthien en terre cuite), (fig. 20, F).

c) Pelile fiole en verre blanchâtre à corps cylindrique et goulot étroit terminé par un orifice évasé à bords ourlés en dedans (fig. 20, E).

d) Vase à liquide en terre cuite jaune pâle très ventru ; col court, anse coudée et pied creux (fig. 20, G).

e) Vase globuleux, de petite taille, en terre grise, tendre, fine, à anse courte (fig. 20, D).

A l'intérieur du ciste :

Tombe 21

a) Urne cinéraire pansue en verre bleu pâle (fig. 21, A).

b) Petit vase rond en terre cuite noirâtre, à pied plein circulaire (fig. 21, B).

c) Outil en fer tige cylindrique prolongée par un manche plat où existent encore les restes d'une plaque de bois fixée par un petit rivet (très fragmenté).

A l'extérieur du ciste :

d) Balsamaire en verre opalin, à corps bulbeux et fond plat (fig. 21, C).

e) Carafon en verre translucide de forme élancée, à col étroit et ouverture évasée ; sillons circulaires autour de la panse et du goulot (fig. 21, D).

f) Verre à boire incomplet à pied arrondi et bords un peu évasés.

g) 4 récipients en verre transparents 1 écuelle et 3 bols du type de ceux rencontrés dans la sépulture 16 (cf. supra), (fig. 21, E, F, G, H).

\section{Tombe 26}

a) Urne cinéraire en verre vert clair (fig. 21, I).

b) "Lacrymatoire" à fond bas, épais, ouverture large et col long (fig. 21, J).

c) Dupondius de FAUSTINE mère (104-141 ap. J.-G.).

A l'avers : FAVSTINA AUGUSTA. - Buste de l'impératrice à droite. Le revers est très fruste.

d) 2 aiguilles en os semblables.

La pièce de monnaie et le "lacrymatoire" se trouvaient dans l'urne parmi les ossements calcinés.

\section{Tombe 28}

Important mobilier funéraire réparti entre l'intérieur et l'extérieur du ciste :

a) Urne cinéraire en verre de même forme que les précédentes (fig. 22, A).

b) Petite ampoule sphérique en verre vert clair à fond un peu aplati, goulot ourlé en dehors, munie de 2 anses dites "delphiniformes" imitation de l'aryballe en terre cuite (forme 33 de MorinJean), (fig. 22, C). 


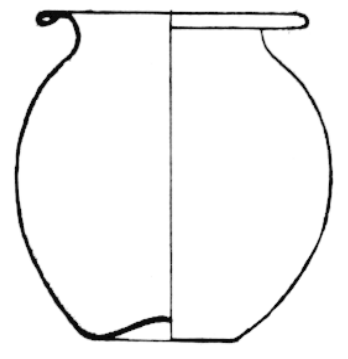

A

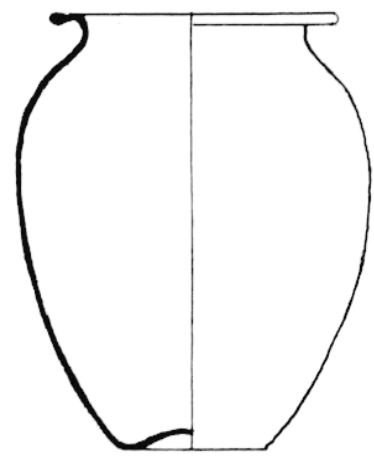

D

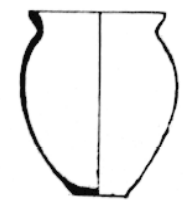

G

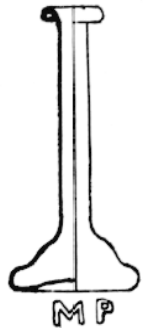

B

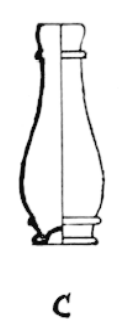

$C$

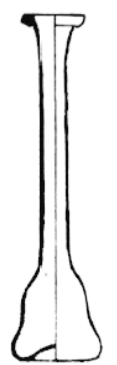

E

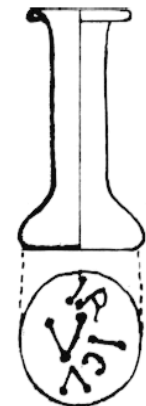

F

$4 \mathrm{~cm}$

H

19. - Tombes $10(\mathrm{~A}, \mathrm{~B}, \mathrm{C})$ et $12(\mathrm{D}, \mathrm{E}, \mathrm{F}, \mathrm{G}, \mathrm{H})$.

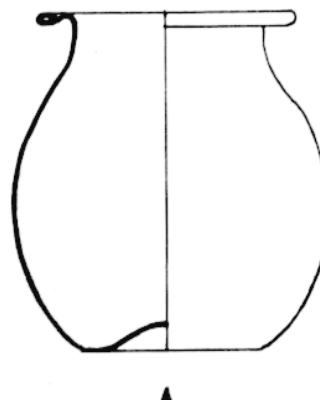

A

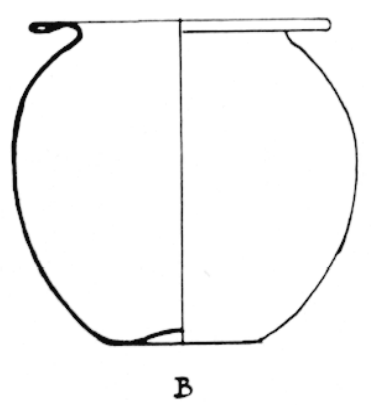

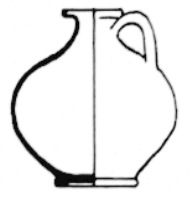

C

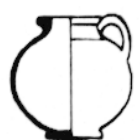

D

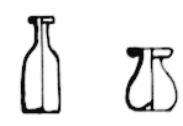

E $\quad F$
Sont

20. - Tombes $18(\mathrm{~A})$ et $20(\mathrm{~B}, \mathrm{C}, \mathrm{D}, \mathrm{E}, \mathrm{F})$.
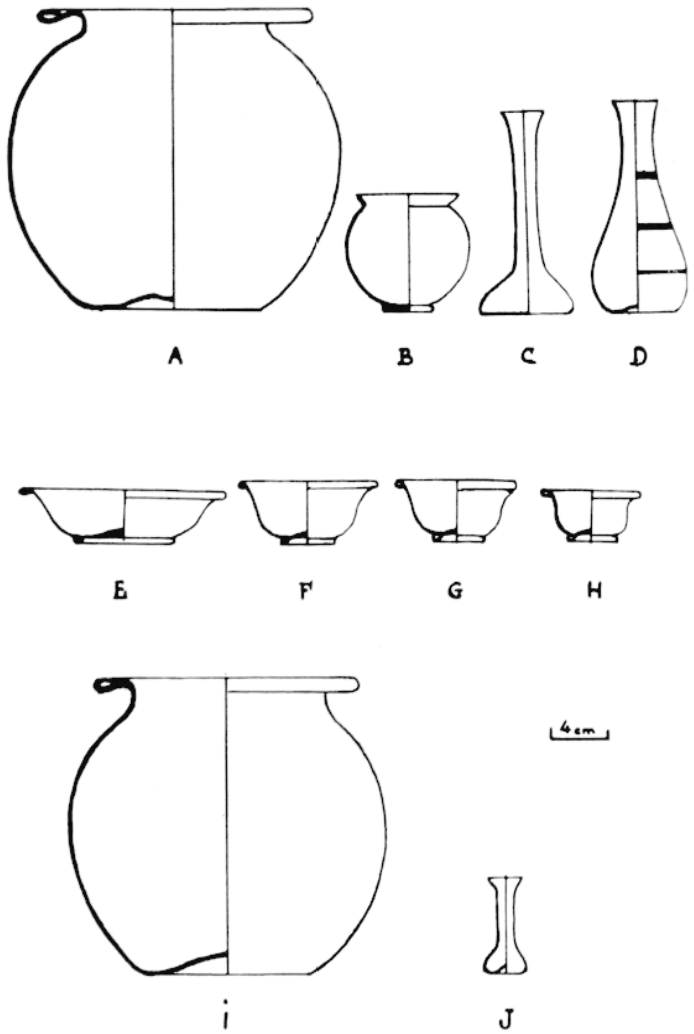

21. - Tombes $21(\mathrm{~A}, \mathrm{~B}, \mathrm{C}, \mathrm{D}, \mathrm{E}, \mathrm{F}, \mathrm{G}, \mathrm{H})$ et $26(\mathrm{I}, \mathrm{J})$.
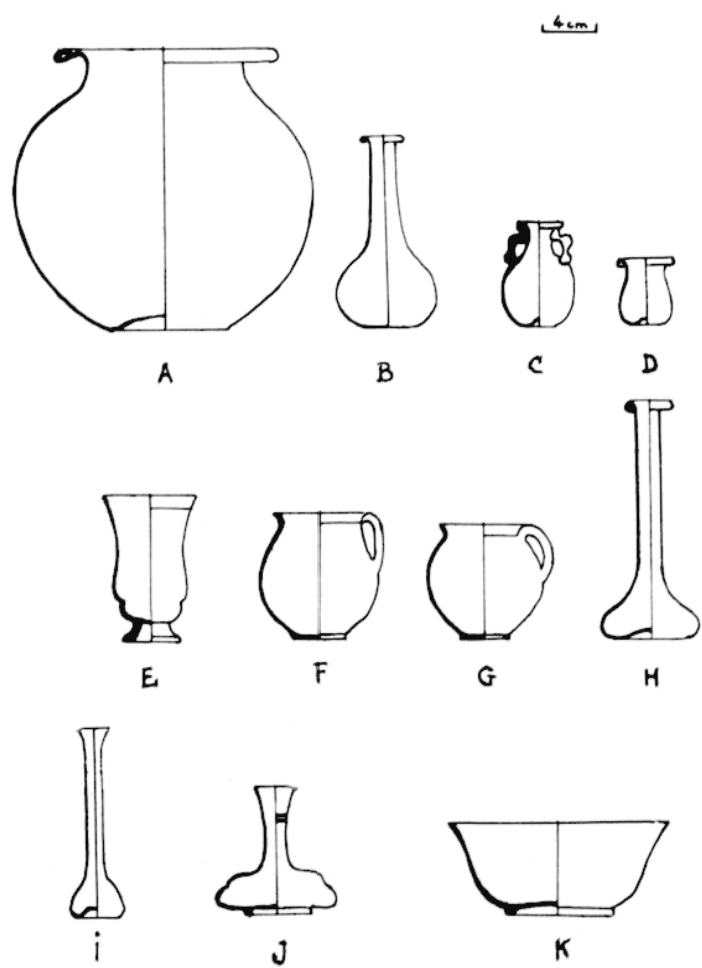

22. - Tombe 28. 
c) Petit " bombylios" de verre identique à celui de la tombe 20 (fig. 22, D).

d) Verre à boire à parois concaves, étranglé à la base qui, épaissie, se termine par un pied tronconique évasé, creux en dessous. Forme se rapprochant du carchesium hellénistique (type 98 de Morin-Jean), (fig. 22, E).

e) Petit vase en poterie brun clair, à anse fine allongée, bords courts un peu évasés avec un léger bourrelet à l'étranglement du col (fig. 22, F).

A còlé du ciste avaient été disposés les récipients suivants :

f) Ampoule en verre bleuté, à panse bulbeuse et col droit (fig. 22, B).

g) Flacon en verre opalin, fin, à embouchure en entonnoir, col cylindrique, panse tronconique surbaissée, tris large et plate ; autour du col, fil de verre enroulé en spirale (fig. $22, \mathrm{~J}$ ).

h) Balsamaire à long col et corps bulbeux aplati, concave en dessous (fig. 22, $\mathrm{H}$ ).

i) Ampoule en verre mat, trop brisée pour qu'on puisse en reconnaitre exactement la forme.

j) Petit balsamaire à panse bulbeuse et bord ourlé, avec marque de verrier très empâtée, illisible, sous le fond. Il contient encore une substance blanche solide semblable à de la craie, qui à l'analyse s'est révélée être "un calcaire naturel impur souillé d'alumine "14 (fig. 22, I).

Linfin, placés sur le couvercle même de la cuve en pierre : $22, \mathrm{~K})$.

k) Écuelle en verre épais translucide imitant la forme Drag. 33 de la céramique italique (fig.

l) Petit vase en terre cuite semblable à celui décrit plus haut (e) de forme un peu plus globuleuse (fig. 22, G).

m) Lampe en argile jaune tendre et friable, type du ${ }_{1}{ }^{\mathrm{e}}$ siècle, à bec un peu allongé, cuvette creuse avec motif ornemental représentant un lion à droite, la tête de face, enserrant dans ses pattes un quadrupède (cheval)? lancé au galop ${ }^{15}$ (fig. 9, B).

Il faut mentionner encore une valve d'huître (ostrea edulis) qui se trouvait parmi le mobilier funéraire.

\section{SÉpultures dans cistes de 'TYPE B}

\section{Tombe 19}

a) Line cinéraire en verre, de forme ovoïde, sans col, à bords ourlés, par exception, extérieurement (fig. 23, A).

b) "Lacrymatoire" à base piriforme, col étranglé à la base et lèvres évasées (f. 21 de MorinJean), (fig. 23, B).

c) Petit vase en forme d'urne en terre commune, bords arrondis, col court et base pleine (fig. $23, \mathrm{C})$.

d) Vase à liquide en poterie jaune à goulot étroit, anse épaisse et pied creux, qui se trouvait a l'extérieur de la tombe (fig. 23, I)).

\section{Tombe 22}

Pas d'urne cinéraire; les ossements avaient été déposés directement dans le ciste. Le mobilier funéraire comprenait :

a) Line écuelle en terre rouge vernissée, lisse (forme Drag. 36), (fig. 23, $\mathrm{H}$ ).

b) Un bol caréné en poterie commune marron clair à patine terreuse ; pied bas circulaire (fig. $23,1)$.

(14) Analyse faite par les services du laboratoire du Musée du Louvre.

(15) Une lampe de la collection Calvet au Musée d'Avignon porte exactement la même décoration (décrite et figurée dans le catalogue des lampes de ce Musée dressé par P. de Brun et S. Gagnière, op. cit., p. 33, no 118 ct p. 34, fig. 118 , pl. VIII). 


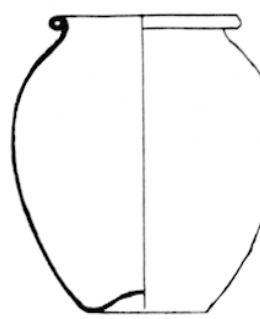

A

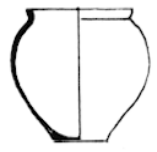

E

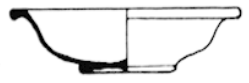

H

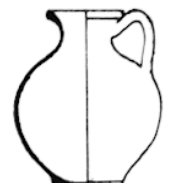

$F$

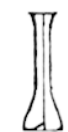

$G$

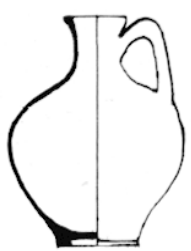

D

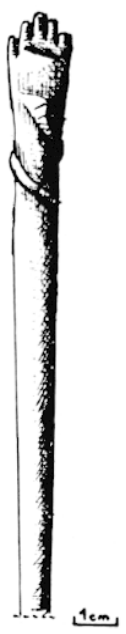

$J$
23. - Tombes $19(\mathrm{~A}, \mathrm{~B}, \mathrm{C}, \mathrm{D})$ et $22(\mathrm{E}, \mathrm{F}, \mathrm{G}, \mathrm{H}, \mathrm{I}, \mathrm{J})$.

c) Un "lacrymaloire" à base tronconique (fig. 23, G).

d) Une épingle à coiffure en os : tige lisse cylindrique s'amincissant en pointe à une extrémité et sculptée de l'autre en forme de main droite ouverte avec bandelette enroulée en spirale autour du poignet (long : $19,4 \mathrm{~cm}$. — larg. en haut : $1,1 \mathrm{~cm}$ ), (fig. 23, J).

e) Les vestiges d'un petit coffrel en bois dont il reste :

104 plaquettes semblables, en os, dégauchies rudimentairement, rectangulaires, entaillées dans un angle $(\mathrm{L}: 3.4 \mathrm{~cm}-\mathrm{l}: 3 \mathrm{~cm}-$ Ep : $0,3 \mathrm{~cm})$, perforées de 2 trous ; dans l'un est enfoncé l'extrémité d'un pied en os chantourné, de $2 \mathrm{~cm}$ de long sur $1,2 \mathrm{~cm}$ de diamètre, l'autre, vide, était destiné à la fixation des plaquettes au fond du coffret au moyen d'une cheville de bois (fig. 24, B).

$2^{\circ}$ Une charnière formée par un axe cylindrique en fer, très oxydé, emmanché dans un tube mobile autour de cet axe (long : $6,3 \mathrm{~cm}$ ), (fig. 24, C).

30 Plusieurs éléments de la serrure, en bronze : plaque d'entrée extérieure discoïde, de $4,2 \mathrm{~cm}$ de diamètre et $0,1 \mathrm{~cm}$ d'épaisseur, traversée en 4 points, opposés 2 à 2 , par un petit clou à tige angulaire et tête ronde, avec ouverture pour l'introduction de la clé, et

3 pièces de son mécanisme intérieur : pène allongé à trous, baguelle plate coudée et petite tige bifide (fig. 24, A, F, D, G).

$4^{\text {o }} 2$ chaînelles, en multiples morceaux, formées par 4 fils de bronze, ténus, tressés, terminées à chaque extrémité par une goupille qui servait à les ancrer aux parois du cofitret ${ }^{16}$ (fig. 24, E et $\mathrm{H}$ ).

f) Un miroir métallique rectangulaire $(\mathrm{L}: 6,5 \mathrm{~cm}-1: 6 \mathrm{~cm}-\mathrm{Ep}: 0,1 \mathrm{~cm})$.

g) Un moyen bronze ou dupondius de VESPASIEN (69-79 ap. J.-C.).

Avers : IMP.CAES. VESPASIAN. AVG. Tête laurée à gauche.

Revers : IVDEA CAPTA. La Judée personnifiée assise sous un palmier, les mains liées au dos, entre $\mathrm{S}$ et $\mathrm{G}$.

Contre le caisson funéraire se trouvaient encore 2 récipients :

h) Une petite urne en poterie grise (fig. 23, E). Dans la terre qui la remplissait a été découvert la coquille, très brisée, d'un æuf de poule bien reconnaissable.

i) Un vase à liquide à goulot étroit et larges bords, anse plate et pied plein (fig. 23, F).

\section{Tombe 30}

a) Urne cinéraire en verre bleu vert qui est une copie très précise de l'olla cineraria habituelle en terre cuite : bords arrondis obliques, col très court, panse surhaussée s'amincissant vers la base terminée par un pied creux (fig. 27, A).

(16) Lne tombe à incinération dans une cuve en pierre découverte en 1960 à St Maurice-sur-Eygues (Drỏme) contenait un coffret du même genre. Il en reste aussi la plaque de serrure, le pène et les deux chaînettes en fils de bronze tressés, avec en plus une petite clé en fer (trouvaille inédite). 


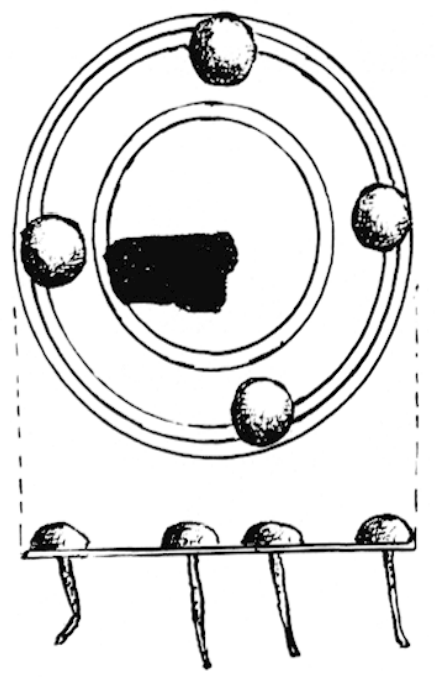

A

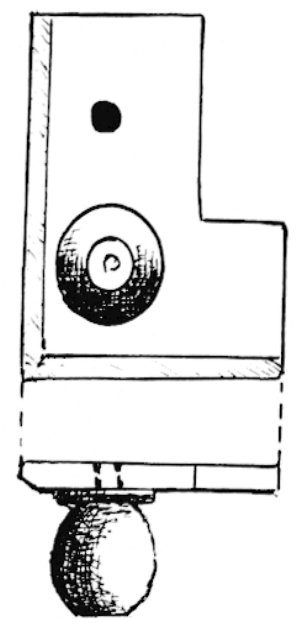

B

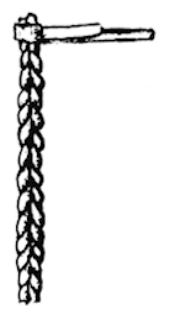

E

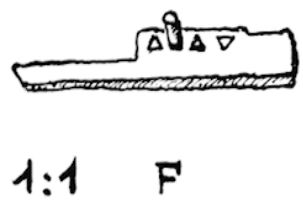

$1: 1 \quad F$ 24. - Tombe 22 : vestiges d'un coffret.

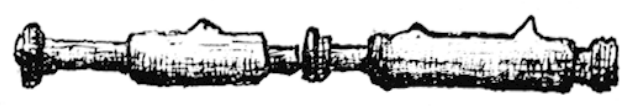

C

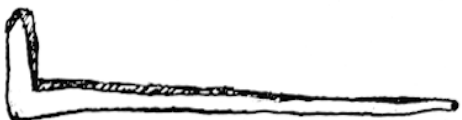

D

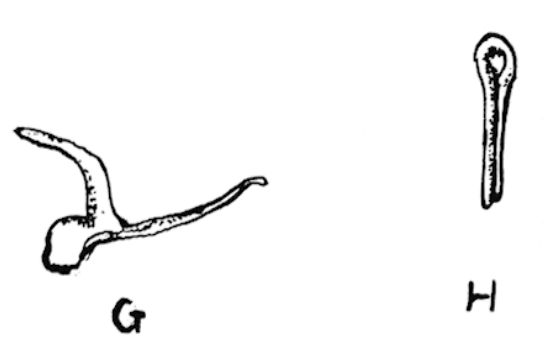

b) "Lacrymaloire" à bords évasés simples, panse conique (fig. 27, B).

c) Écuelle en terre rouge vernissée, rebord orné de feuilles d'eau barbotinées sinistrogyres (f. Drag. 36), (fig. 27, G).

d) Petit vase en poterie fine jaunâtre tendre muni de 2 anses ovales opposées formées de 2 petits boudins jumelés verticaux, lèvres à bourrelet ténu, panse ornée d'un quadrillage formé d'éléments barbotinés disposés en quinconce, base annulaire simple ${ }^{17}$ (fig. 27, F).

e) Vase peu élevé en terre cuite ordinaire, forme d'urne en réduction (fig. 27, D).

f) Lampe en argile marron clair à bec rond flanqué de volutes. Le dessus est orné d'une tympanistria: femme drapée vue de trois quarts coiffée d'une sorte de turban à voile descendant de chaque côté du visage, tenant un tympanum ou tambour de basque de la main gauche, la droite étant posée sur la hanche (forme Dressel 11 - lampe de la fin du I ${ }^{\text {er }}$ siècle - type B) ${ }^{18}$ (fig. 8, F).

g) Jeton discoïde en verre verdâtre brillant, percé au centre (D:2,1 cm - Ep. :0,7 cm).

h) Vase à liquide pansu en terre jaune à pied large et anse courte coudée, qui se trouvait en dehors de la tombe (fig. $27, \mathrm{E}$ ).

(17) Ce type de vase, également trouvé à Glanum, a été classé par P. de Brun dans la poterie fine, dite de fantaisie du ier siècle ap. J.-C. Noles sur la céramique anlique de Sl-Rémy-de-Provence (B.-du-R.), $2^{2}$ partie. Succession des divers types, Rhodania, 1931, no 1566, pl. II, fig. 1. Un récipient identique a été découvert en 1956 près de Vidauban (Var) dans un dépót d'incinération cf. R. Boy ER, Récentes découvertes archéologiques aux Blais (Var). Cahiers ligures de préhistoire et d'archéologie, 8, 1959, p. 94, fig. 6, no 1.

(18) Même sujet figuré sur une lampe du Musée Calvet, provenant de Vaison. P. de Brun et S. GaGnière, op. cit., p. 28, pl. VI, n 78 . 

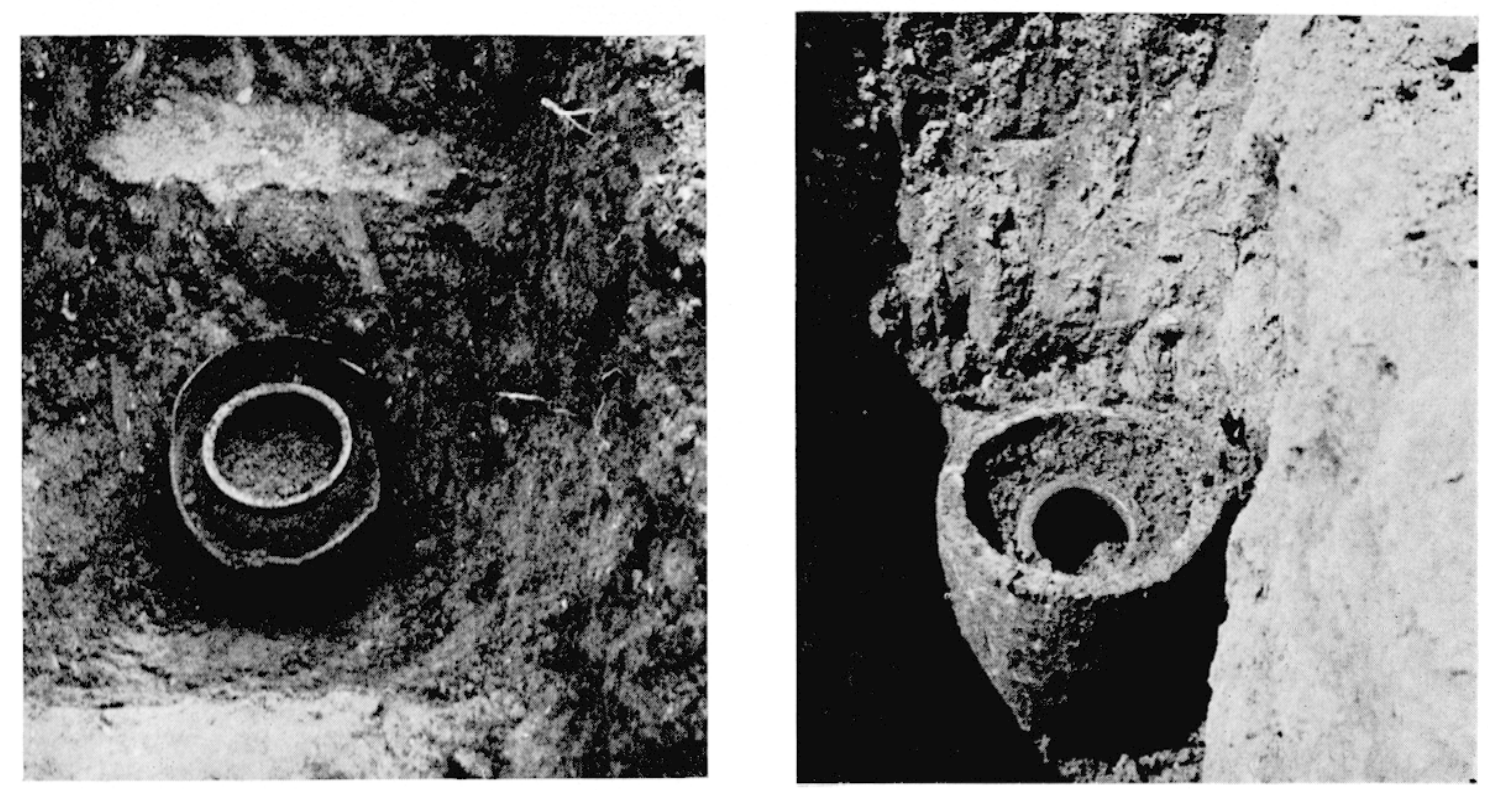

25. - Sépultures dans amphore décapitée (tombe 24) et ciste cylindro-conique (tombe 28), avec urne cinéraire.
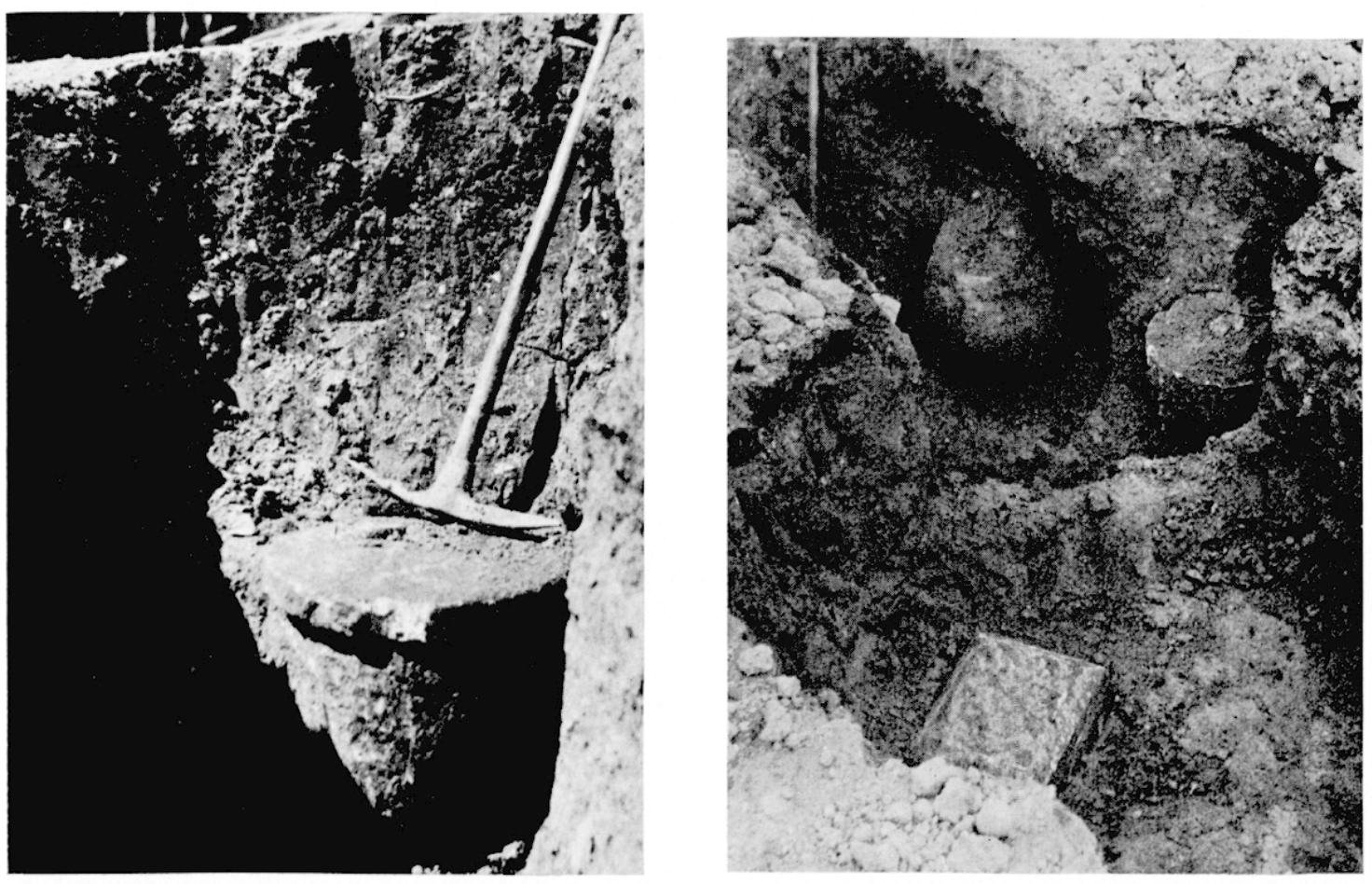

26. - Sépultures groupées : tombes 26 et 27 , tombes $17,18,19$. 


\section{Tombe 33}

a) Urne cinéraire en verre, de petite taille, trop morcelée pour être reconstituée.

b) Verre à boire de teinte claire, à profil galbé évasé au bord, fond plat ${ }^{19}$; parois ornée de 3 petits sillons vers le haut (f. 105 de Morin-Jean), (fig. 28, A).

c) "Lacrymaloire " à col resserré à la base du col, type courant (fig. 28, B).

d) Écuelle en terre rouge vernissée. Lettre $\Lambda$ gravée à la pointe sèche sous le pied (f. Drag. 36), (fig. 28, C).

e) Petite urne en terre grisâtre (fig. 28, D).

f) 6 jelons plats en os dont 3 lisses et 3 ornés de plusieurs cercles concentriques sur une face (D : $2,7 \mathrm{~cm}-\mathrm{Ep}: 0,3 \mathrm{~cm}$ ).

g) Perle còtelée en pâte de verre verdâtre, opaque. (H : 1,2 cm - D : 1,5 cm), (fig. $28, \mathrm{~K})$.

h) Intaille minuscule, ovale, en verre clair orné d'un motif gravé paraissant représenter un vase à 2 anses d'où se développe une digitation trifide qui n'est pas sans quelque ressemblance avec une fleur de lys stylisée. (L : $0,7 \mathrm{~cm}-1: 0,5 \mathrm{~cm}$ ), (fig. 28, L).

i) Clochette en bronze, courte, ouverte en haut avec anneau de suspension et battant mobile en fer (fig. 28, J).

Un an après la découverte de cet ensemble de 33 tombes, au mois de février 1959 , en creusant le sol pour la construction d'une cuve à l'intérieur même de l'usine de fruits confits Louis Piton, une amphore en bon état du type I (à pied évidé et à panse surhaussée) a été mise au jour.

De l'intérieur ont été retirés les objets suivants :

a) Une urne cinéraire en verre contenant quelques ossements calcinés de petite taille appartenant $\dot{a}$ un enfant mort en très bas âge (fig. $28, \mathrm{E}$ ).

b) Un "lacrymatoire " à fond rond et col très allongé ; trouvé dans l'urne parmi les ossements (fig. 28, G).

c) Une urnetle en terre cuite grise commune (fig. $28, \mathrm{H}$ ).

d) Un bol à panse angulaire et rebord horizontal ; même type de poterie que le récipient précédent (fig. 28, F).

e) Un petit vase à liquide en terre marron clair à panse ovoïde surmontée d'un col cylindrique terminé par un rebord plat; anse étroite arrondie verticale et pied en forme de bourrelet circulaire. Ce qui fait l'originalité de ce récipient c'est l'existence, en haut de la panse d'une courte tubulure conique à pointe effilée horizontale perforée obliquement permettant l'écoulement du liquide autrement que par le goulot. Il s'agit certainement d'un biberon d'enfant ${ }^{20}$. Contenance exacte : 16,5 cl

(19) Le Musée Calvet possède dans sa collection de verrerie gallo-romaine 2 verres à boire semblables : l'un provient d'Apt où il été trouvé dans un tombeau avec une urne en verre en 1848, l'autre était aussi dans un tombeau avec urne en verre découvert dans le terroir de Bonnieux, en construisant le chemin vicinal no 6 qui conduit au Pont Julien, quartier dit de Lovière, en 1855) (Catalogue manuscrit du Musée Calvet, Antiquités égyptiennes grecques et romaines, p. $475,99 \mathrm{~A}$ et $99 \mathrm{~B}$ ).

(20) Cette dénomination a été contestée par certains archéologues, notamment par Morin-Jean, qui figure cette forme particulière de récipient dans son ouvrage sur la verrerie en Gaule, car elle existait aussi en verre (forme 52, pp. 109-110). Il est pourtant facile en remplissant d'un liquide quelconque le récipient jusqu'au bord de constater que celui-ci ne s'écoule pas par la tubulure de la paroi à cause de son faible diamètre mais si on exerce une succion, le liquide sort sans difficulté. La présence de ce récipient, peu courant, dans une tombe d'enfant viendrait confirmer la véracité de cette attribution. Des biberons en terre cuite ont été trauvés à Vaison dans des tombeaux lors des fouilles de 1841. Cinq exemplaires figurent dans les vitrines du Musée Calvet (d'après J. Saltes, Vaison dans l'Anliquilé, II, Calalogue des objets romains trouvés à Vaison el dans son lerriloire, 1926, p. 364, no 1134 à 1137 et fig. 9, pl. LXXV du recueil documentaire illustré). Au Musée de Vichy on peut voir aussi trois biberons trouvés dans le sous-sol des Aquae Calidae. Leur contenance et leur taille sont voisines du nòtre; l'anse est aussi "dans un plan perpendiculaire à celui du déversoir latéral ", Dr Morlet, Vichy gallo-romain, p. 59 (fig. 27). 


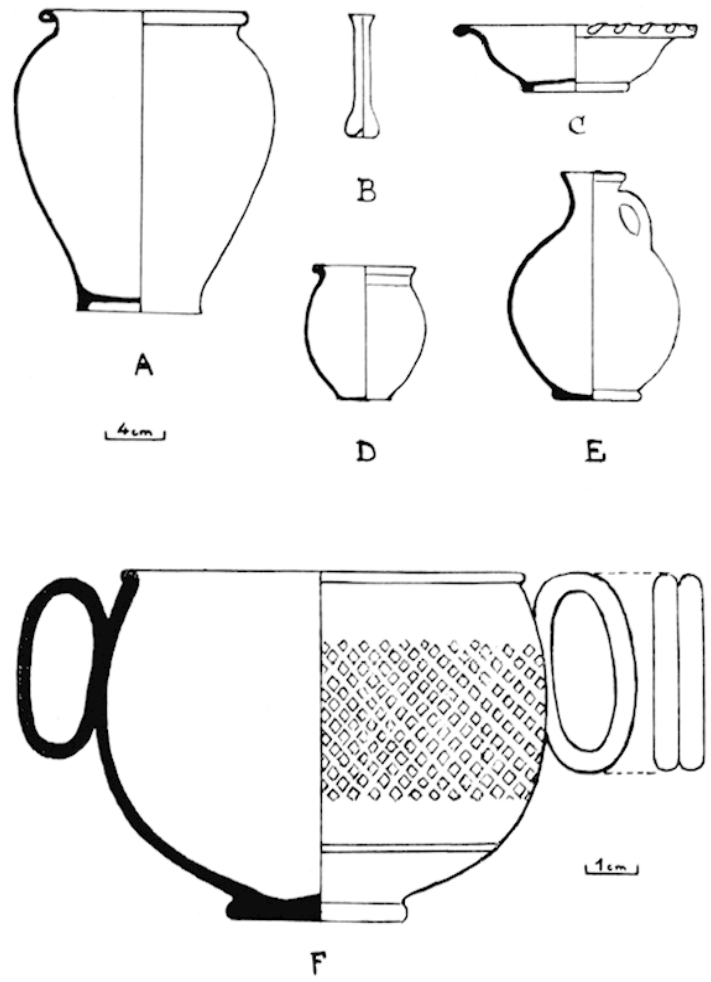

27. - Tombe 30 .

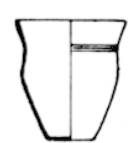

A

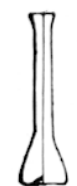

B

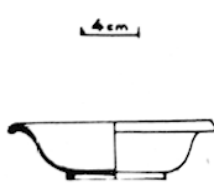

c

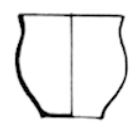

D

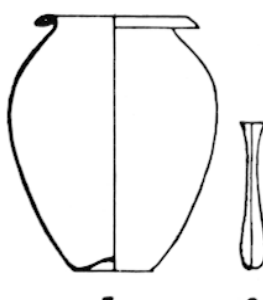

E
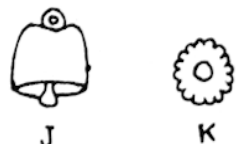

K

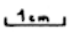

28. - Tombes $33(\mathrm{~A}, \mathrm{~B}, \mathrm{C}, \mathrm{D}, \mathrm{J}, \mathrm{K}, \mathrm{L})$ et $34(\mathrm{E}, \mathrm{F}, \mathrm{G}, \mathrm{H}, \mathrm{I}, \mathrm{M})$

(H : 11,4 cm - DO :3,3 cm - DM : 7,5 cm - DB :3,4 cm - D. du tuyau : 0,2 cm), (fig. $28, \mathrm{I})$.

f) Une lampe en terre cuite orangée de forme très régulière avec grand trou central sur le dessus, 2 boutons sur les bords et canal médian interrompu au bec où il se termine par un petit orifice d'aération. Marque du potier FORTIS sur le fond. Type de la vallée du Pô, forme Dressel 5 B (fig. 8, E).

g) Une clochelle en bronze à ouverture circulaire munie d'un anneau fixe pentagonal à sa partie supérieure ( $\mathrm{H}: 3 \mathrm{~cm}-\mathrm{D}: 2,4 \mathrm{~cm}$ ) (à rapprocher de celle trouvée dans la tombe 33 qui était aussi la sépulture d'un enfant) ${ }^{21}$, (fig. 28, M).

h) 2 perles semblables en pâte de verre bleu ciel, globuleuses, à surface côtelée ( $\mathrm{H}: 1,3 \mathrm{~cm}-$ D : $1,4 \mathrm{~cm})$.

i) 17 perles cylindriques en substance fibreuse vitrifiée $e^{22}(\mathrm{~L}: 0,8 \mathrm{~cm}-\mathrm{D}: 0,5 \mathrm{~cm})$.

j) Un ressort en bronze à 7 spires (L : 2,2 $\mathrm{cm}-\mathrm{D}: 0,8 \mathrm{~cm}$ ).

(21) Des clochettes absolument semblables comme forme et taille garnissent un buste de Mercure en bronze découvert à Orange et conservé au Cabinet des médailles de la Bibliothèque nationale (figuré dans le Manuel d'Archéologie de Cagivat et Cinapot, II, p. 194, fig. 446). Répandues comme ornements garnissant des bracelets et des colliers, l'auteur interprète leur existence comme amulette "propre à couvrir les bruits de mauvais augure ".

(22) D'après leur analyse, faite au laboratoire du Musée du Louvre, ces perles présentent 2 couches d'émail : l'une extérieure, en émail vert faiblement coloré à l'oxyde de fer, l'autre, intérieure, en émail jaune montrant des parties charbonneuses, ce qui permet de conclure que "ces perles semblent avoir été faites de 2 couches d'émail en employant comme matrice une matière organique (nœud de paille ou graine ?) qui se serait calcinée pendant la vitrification et dont l'émail intérieur aurait gardé l'empreinte" 


\section{ConcLesions}

Cet ensemble de sépultures fait partie d'un cimetière gallo-romain à incinération situé, selon. la coutume, hors de la cité et le long d'une route y accédant "afin que les passants puissent se rappeler et honorer les morts ${ }^{23}$. Les remaniements du terrain, dus aux travaux modernes et aux constructions, ne nous ont pas permis d'en constater les prolongements aux abords immédiats de l'aire explorée, mais des trouvailles sporadiques de tombes dans tout ce quartier ${ }^{24}$ ne laissent pas le moindre doute sur l'importance de cette nécropole. Le mobilier est présenté au musée d'Apt (fig. 29).

Ces découvertes nous conduisent à formuler brièvement quelques remarques. D'abord constatons qu'aucun ordre géométrique n'a présidé à la disposition des tombes, placées sans tracé précis ni alignement, à des distances variables les unes des autres, le rapprochement de certaines pouvant faire penser à un groupement de sépultures d'une même famille. A noter l'absence totale de stèles ou cippes funéraires; il s'agit certainement d'un champ de repos pour gens de condition modeste. On peut encore souligner la variété dans le choix du tombeau lui-même : 2 sortes de cistes et 2 types d'amphores sur la panse desquelles sont. quelquefois gravés les noms des défunts : IIRMIIS, PASS (iénus) et CAM (panus) (?). Pas d'uniformité non plus dans le mobilier funéraire dont une partic a été souvent disposée, faute de place, à l'extérieur du caisson ou de l'amphore. Prédominance très marquée des urnes cinéraires en verre sur celles en terre cuite ; abondance et diversité de formes parmi les récipients en verre : ceux rencontrés le plus souvent sont les balsamaires et les "lacrymatoires". Rareté de la pièce de monnaie pour le "passage du Styx " ct pratique exceptionnelle du repas funéraire (œuf de la tombe 22 et huître de la sépulture 28). En somme, variété due à des raisons purement subjectives : croyance religieuse, fortune et sentiments familiaux.

Quant à la période à laquelle on peut faire remonter ce cimetière, un critère d'abord : le rite exclusif de la crémation, pratiqué uniquement pendant les deux premiers siècles de l'époque impériale ainsi que l'indiquent les nombreuses trouvailles faites dans notre région ${ }^{25}$. Ensuite les monnaies qui, quoiqu'on ne puisse leur attribuer une valeur de datation absolue puisque l'on a observé de nombreux exemples du dépôt de monnaies n'ayant plus qu'un cours fiduciaire, donnent cependant des points de repère intéressants. Ainsi nous avons 2 empereurs : Vespasien (69-79) et Domitien (81-96) de la $2^{\mathrm{e}}$ moitié du $\mathrm{I}^{\mathrm{er}}$ siècle, un empereur : Trajan (98-117) et une impératrice Favstine I (104-131) de la $1^{\text {re }}$ moitié du second. Les lampes funéraires sont de 3 types : $1^{0} A$ volutes ( 2 exemplaires l'un accompagnant un dupondius de Domitien) dont la forme disparait totalement au début $d u{ }_{1}{ }^{\mathrm{e}}$ siècle. $2^{\circ}$ type de la vallée du Pô (4 exemplaires). Une lampe à canal fermé, signée FORTIS (dernière tombe) serait d'après le professeur Nino Lamboglia une forme

23) Albert Grenier, Manuel... Deuxième partie : L'Archéologie du sol; les roules, p. 213.

(24) Notamment en 1955, 4 sépultures dans la propriété de Mr Julien, maire de Saıcivos, à une centaine de mètres à l'Est de l'Ĺsine Piton (cf. A. Dumoulin, Gallia, op. cil., p. 266 à 210).

(25) Par exemple à Vaison, Apt, Cavaillon, Robion, Goult, Gordes, Rustrel, Sannes, etc... et récemment à Malaucène, Ste Cécile-les-Vignes et Visan. (Gallia, XIV-1956, pp. 253 à 256). 

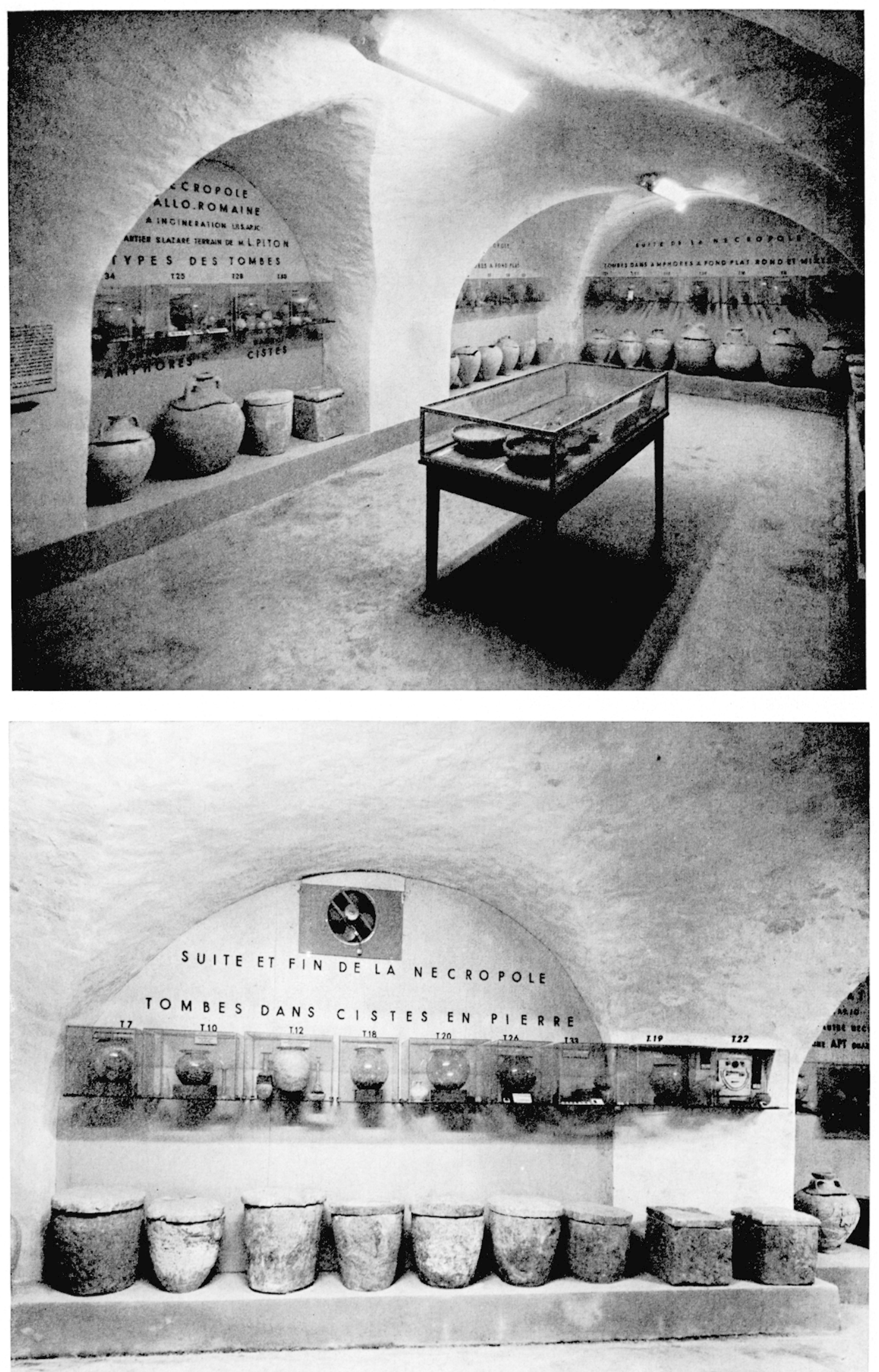

29. - Présentation des objets au Musée d'Apt. 
"postérieure à l'an 60 et antérieure à l'an 100 "26. Les 3 autres, à canal ouvert, 2 de FORTIS, 1 signée PVLLI, seraient d'après le même auteur exclusivement du $\mathrm{II}^{\mathrm{e}}$ siècle. $3^{\circ}$ type à bec rond simple séparé de la cuvette par une incision horizontale ( 3 exemplaires) également $\mathrm{du}$ II $^{\mathbf{e}}$ siècle. Même date pour les amphores à pied. Les ustensiles en poterie commune : écuelles, bols, urnettes, vases à liquide, sont aussi des formes du Haut-empire ${ }^{27}$. La poterie rouge vernissée est assez peu représentée ; pas de vases ornés ; les récipients déposés dans les tombes, à teinte particulièrement mate et à formes molles, paraissent être des produits d'une offine locale imitant les types courants de la Graufesenque. La "sigillée claire " n'a donné qu'un seul vase (tombe 23) comparable à la forme 19 de la série A, daté du ${ }_{11}{ }^{\mathrm{e}}$ siècle $^{28}$. La verrerie se rapporte entièrement au romain I de Morin-Jean (de l'époque d'Auguste à la fin du règne de Commode 192 ap. J.-C.).

Les quelques inscriptions gravées sur poteries ne nous apprennent rien de très précis, pas plus l'absence du $\mathrm{H}$ devant ERMES, que la graphie II pour E, mais la forme du A à barre oblique et celle du $\mathrm{M}$, sont bien caractéristiques du I $^{\text {er }}$ siècle $^{29}$. On peut donc considérer que cette nécropole a été utilisée vers la fin du ier siècle et pendant la plus grande partie du ${ } \mathrm{e}^{\mathrm{e}}$.

André Dumovlin.

(26) Cité dans : A. Bentrax, Apuntes sobre eronologia céramica, Publicaliones del seminario de arquenlogia y numismatica Aragonesa, 1952, p. 88 et 89.

(27) On les retrouve à la nécropole de Sannes (Vaucluse, A. Dumoc ın, Gallia, op. cil).

(28) Nino Lambogina, Nuove osservazioni sulla "lerra sigillala chiara", Tipi A et B, Rivisla di studi liguri, XXIV, $1958, n^{\circ} 3-4$.

(29) Lettres semblables parmi les nombreux graffitis de La Graufesenque (cf. F. HIsrmet, La Graufesenque (Condalomago), $2^{\mathrm{e}}$ partie, planches, in fine) et aussi sur poterie rouge à Glanum (H. Rolland, Inscriplions anliques de Glanum (Sl-Remy-de-Provence), Gallia, II, 1944, p. 198, no 88). 